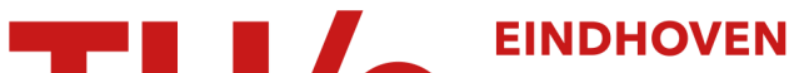 \\ UNIVERSITY OF \\ TECHNOLOGY
}

\section{Schedule-extended synchronous dataflow graphs}

Citation for published version (APA):

Damavandpeyma, M., Stuijk, S., Basten, T., Geilen, M. C. W., \& Corporaal, H. (2013). Schedule-extended synchronous dataflow graphs. (ES reports; Vol. 2013-01). Eindhoven University of Technology.

Document status and date:

Published: 01/01/2013

\section{Document Version:}

Publisher's PDF, also known as Version of Record (includes final page, issue and volume numbers)

\section{Please check the document version of this publication:}

- A submitted manuscript is the version of the article upon submission and before peer-review. There can be important differences between the submitted version and the official published version of record. People interested in the research are advised to contact the author for the final version of the publication, or visit the $\mathrm{DOI}$ to the publisher's website.

- The final author version and the galley proof are versions of the publication after peer review.

- The final published version features the final layout of the paper including the volume, issue and page numbers.

Link to publication

\section{General rights}

Copyright and moral rights for the publications made accessible in the public portal are retained by the authors and/or other copyright owners and it is a condition of accessing publications that users recognise and abide by the legal requirements associated with these rights.

- Users may download and print one copy of any publication from the public portal for the purpose of private study or research.

- You may not further distribute the material or use it for any profit-making activity or commercial gain

- You may freely distribute the URL identifying the publication in the public portal.

If the publication is distributed under the terms of Article 25fa of the Dutch Copyright Act, indicated by the "Taverne" license above, please follow below link for the End User Agreement:

www.tue.nl/taverne

Take down policy

If you believe that this document breaches copyright please contact us at:

openaccess@tue.nl

providing details and we will investigate your claim. 


\section{Schedule-Extended Synchronous Dataflow Graphs}

Morteza Damavandpeyma, Sander Stuijk, Twan Basten, Marc Geilen and Henk Corporaal

This report is an extension of the following journal paper to be published. It adds the proofs omitted from the publication. If you want to cite this report, please refer to the paper instead.

M. Damavandpeyma, S. Stuijk, T. Basten, M.C.W. Geilen and H. Corporaal, "Schedule-Extended Synchronous Dataflow Graphs”. IEEE Transactions on Computer-Aided Design of Integrated Circuits and Systems, 2013.

\section{ES Reports}

ISSN 1574-9517

ESR-2013-01

17 May 2013

Eindhoven University of Technology

Department of Electrical Engineering Electronic Systems 
Copyright (c) 2013 IEEE. Personal use of this material is permitted. However, permission to use this material for any other purposes must be obtained from the IEEE by sending an email to pubs-permissions@ieee.org.

http://www.es.ele.tue.nl/esreports

esreports@es.ele.tue.nl

Eindhoven University of Technology

Department of Electrical Engineering

Electronic Systems

PO Box 513

NL-5600 MB Eindhoven

The Netherlands 


\title{
Schedule-Extended Synchronous Dataflow Graphs
}

\author{
Morteza Damavandpeyma, Student Member, IEEE, Sander Stuijk, Twan Basten, Senior Member, IEEE, \\ Marc Geilen, Member, IEEE, and Henk Corporaal, Member, IEEE
}

\begin{abstract}
Synchronous dataflow graphs (SDFGs) are used extensively to model streaming applications. An SDFG can be extended with scheduling decisions, allowing SDFG analysis to obtain properties like throughput or buffer sizes for the scheduled graphs. Analysis times depend strongly on the size of the SDFG. SDFGs can be statically scheduled using static-order schedules. The only generally applicable technique to model a static-order schedule in an SDFG is to convert it to a homogeneous SDFG (HSDFG). This may lead to an exponential increase in the size of the graph and to sub-optimal analysis results (e.g., for buffer sizes in multi-processors). We present techniques to model two types of static-order schedules, i.e., periodic schedules and periodic single appearance schedules, directly in an SDFG. Experiments show that both techniques produce more compact graphs compared to the technique that relies on a conversion to an HSDFG. This results in reduced analysis times for performance properties and tighter resource requirements.
\end{abstract}

Index Terms-Synchronous dataflow graphs, periodic schedules, single appearance schedules, schedule modeling.

\section{INTRODUCTION}

Synchronous dataflow graphs (SDFGs) are widely used to model digital signal processing (DSP) and multimedia applications [1]-[4]. Model-based design-flows (e.g., [1], [5]-[8]) model binding and scheduling decisions into the SDFG. This enables the analysis of performance properties (e.g, throughput [9]) or resource requirements (e.g., buffer sizes [10]) under resource constraints. Figure 1 shows an example of an SDFG with four actors and three channels. An essential property of SDFGs is that every time an actor fires (executes) it consumes a fixed number of tokens from its input edges and produces a fixed number of tokens on its output edges. These numbers are called the rates (indicated next to the channel ends when the rates are larger than 1). The fixed port rates make it possible to statically schedule SDFGs.

Many SDFG analysis algorithms, e.g., throughput calculation or buffer sizing, are straightforward when a single processor platform is used. For instance, the buffer sizes can be determined by executing the SDFG according to a given schedule. However, in a multi-processor environment, SDFG analysis algorithms are not trivial because of the interprocessor communication, amongst other reasons. An SDFG can be bound to a multi-processor platform. Each processor in the platform executes a set of actors from the SDFG; the firings of actors bound to a processor are required to be sequentialized. For this purpose, a finite periodic schedule can

The authors are with the Department of Electrical Engineering, Eindhoven University of Technology, Eindhoven, The Netherlands (e-mail:\{m.damavandpeyma, s.stuijk, a.a.basten, m.c.w.geilen, h.corporaal\}@tue.nl).

T. Basten is also with TNO, Embedded Systems Innovation, Eindhoven, The Netherlands.

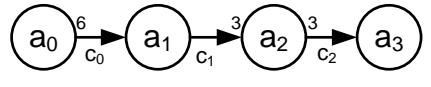

Fig. 1. An example SDFG.

be constructed. Such a schedule is called a periodic staticorder schedule (PSOS). PSOSs only specify the firing order of actors. This separates them from fully static schedules, which determine absolute start times of actors (e.g., schedules generated using the technique of [11]). Traditionally, for DSP software synthesis, a sub-set of all periodic static-order schedules is considered. This sub-set contains so-called single appearance schedules (SAS) [1]. In a SAS, the functional code of the actors is included in a nested loop structure such that each piece of code occurs only once. This minimizes the code size potentially at the cost of additional buffer memory needed to implement the channels. A model-based design-flow usually uses PSOSs (or a sub-set of PSOSs such as SASs) for an application modeled with an SDFG. In this way timing (throughput) and memory usage (buffers) can be analyzed.

There is only one technique [12] known to model PSOSs in an SDFG. This technique requires a conversion of an SDFG to a so-called homogeneous SDFG (HSDFG) in which all rates are one [2]. Figure 2 (without the colored edges) shows the equivalent HSDFG of the SDFG in Figure 1. The technique of [12] sequentializes the actor firings by inserting a channel between each pair of consecutive actors in a schedule. At the end of a schedule, it adds a channel with one initial token from the last to the first actor in the schedule. This ensures an indefinite execution of the graph according to the schedule. To model PSOSs $s_{0}=\left\langle a_{0}\left(a_{2}\right)^{2}\right\rangle^{*}$ and $s_{1}=\left\langle\left(a_{1}\right)^{5}\left(a_{3}\right)^{3} a_{1}\left(a_{3}\right)^{3}\right\rangle^{*}$, the technique of [12] adds in total 15 channels to the HSDFG of the example graph (the green edges for $s_{0}$ and the blue edges for $s_{1}$ in Figure 2). For example, $s_{0}$ indicates an indefinite sequence of one firing of $a_{0}$ followed by two firings of $a_{2}$. This order is enforced in the HSDFG of Figure 2 by the green edges between the actors $a_{0 \_}, a_{2 \_1}$, and $a_{2 \_}$.

The SDFG to HSDFG conversion can lead to an exponential increase in the size of the graph. For example, such a conversion for an H.263 decoder [10] (with QCIF resolution) increases the graph size from 4 actors to 1190 actors. Note that the number of actors in the resulting HSDFG highly depends on how the application is modeled in the original SDFG. The run-time of SDFG analysis algorithms depends amongst others on the size of the graph. As a result, the run-time of many SDFG analysis algorithms may increase drastically when modeling PSOSs in the graph using the technique from [12]. For example, the buffer sizing algorithm from [10] takes less 


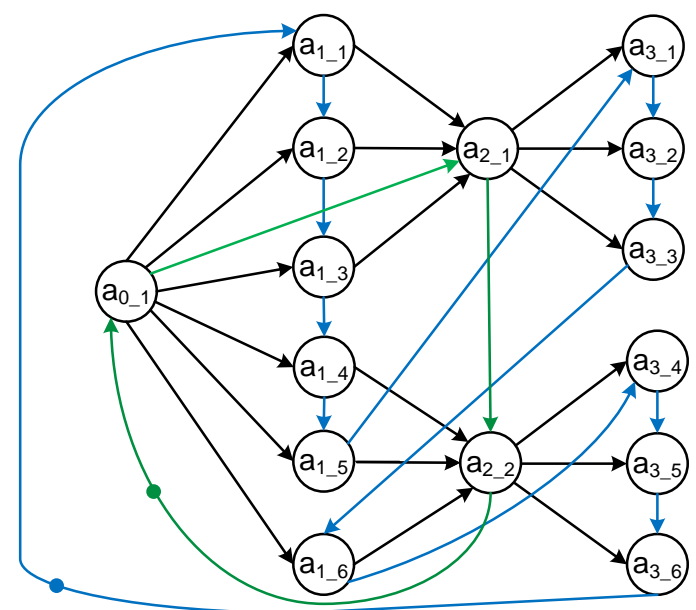

Fig. 2. PSOSs $s_{0}=\left\langle a_{0}\left(a_{2}\right)^{2}\right\rangle^{*}$ and $s_{1}=\left\langle\left(a_{1}\right)^{5}\left(a_{3}\right)^{3} a_{1}\left(a_{3}\right)^{3}\right\rangle^{*}$ modeled in the SDFG of Figure 1 using the technique from [12]; each $a_{i_{j} j}$ actor in the HSDFG is an instance of SDFG actor $a_{i}$.

than $1 \mathrm{~ms}$ on the SDFG of an H.263 decoder. Modeling a schedule into this SDFG using the technique from [12], the same analysis lasts $1330 \mathrm{~ms}$. SDFG analysis algorithms are usually repeated more than once in an iterative designflow. As an example, for the SDFG of an H.263 decoder, the design-flow from [6] performs 8 throughput calculations on the SDFG to obtain the desired binding. Hence, it is vital to maintain a compact schedule-extended graph, i.e., a graph in which schedules are modeled explicitly, to provide a fast and practical design flow. There is a second drawback to the technique from [12]. The original graph structure is lost due to the conversion to an HSDFG. A single channel in an SDFG corresponds to a set of channels in the HSDFG. In Figure 2, for example, the six edges between actor $a_{0 \_1}$ and the $a_{1 \_j}$ actors correspond to the single edge between $a_{0}$ and $a_{1}$ in the SDFG of Figure 1. As a result, common buffer sizing techniques cannot find the minimal buffer size for the original SDFG. The H.263 decoder buffer sizes are for example overestimated by $49 \%$ when applying the technique of [10] to the HSDFG.

A novel technique is needed to model PSOSs in an SDFG. This technique should limit the increase in the number of actors such that analysis times do not increase too much when analyzing the SDFG with its schedules. The technique should also preserve the original graph structure as this enables accurate analysis of graph properties such as buffer sizes. In [13], we presented a schedule modeling technique, called DSM, to model any PSOS directly in an SDFG. In this paper, DSM is discussed in more detail. In addition, a second schedule modeling technique, called SASM, is introduced that is limited to SASs, but that results in more compact models compared to the first technique when modeling SASs. Correctness of the two approaches is formalized. Extensive experiments are carried out for evaluation purposes.

DSM and SASM can be used in any model-based designflow that models PSOSs into the SDFG (e.g., [1], [5]-[8]). Conversion to an HSDFG may be inevitable at some steps of a design trajectory. For example, multi-processor scheduling may require such conversions, although some techniques exist that can find schedules for SDFGs without any conversion to HSDFGs. For example, the technique presented in [14] solves the buffer sizing and scheduling problems simultaneously at the SDFG level. It is not the conversion from SDFG to HSDFG itself that is problematic though. The problem is that analyses or optimizations on large HSDFGs may be time consuming (e.g. throughput analysis) or inaccurate (e.g. buffer sizing). With our techniques, obtained schedules can be annotated back to the original SDFG; hence, the later analysis and optimization can be performed on the scheduleextended SDFG. Besides the already mentioned analyses, also for example dynamic voltage scaling can be directly applied to a schedule-extended SDFG model of an application mapped to a multi-processor platform [15]. Note that code generation is another step which requires an SDFG to HSDFG conversion; this conversion can be delayed until all (or most of the) prior analyses are carried out on the SDFG. Ultimately, the proposed techniques may save significant amounts of analysis time in a multi-processor design flow and they may lead to more accurate results.

The remainder of the paper is structured as follows. The next section discusses related work. Section III introduces SDFGs. Section IV formalizes SDFG schedules. Sections V and VI present our techniques to model PSOSs and SASs in an SDFG. Section VII contains the theorems related to the correctness of the presented techniques. We evaluate our technique by applying it to several realistic applications in Section VIII. Section IX concludes.

\section{RELATED WORK}

The technique from [12] is the only available technique to model PSOSs in an SDFG, through a conversion to an HSDFG. As already explained, this technique may result in a long run-time for analysis algorithms and/or inaccurate results from these algorithms. Our techniques alleviate both shortcomings of the technique from [12].

The work in [16] models the effect of a budget scheduler or preemptive TDMA scheduler on the temporal behavior of the SDFG, either by computing an accurate worst-case response time or, more precisely, by introducing additional actors to model the timing impact as a latency-rate model. In contrast, for non-preemptive schedules, such as PSOSs, we focus on the ordering of actor firings; their execution time remains the same. We force an SDFG to follow the PSOSs selected for each processor. This allows SDFG analysis to obtain properties like throughput or buffer sizes for the scheduled SDFG. This is also true for the models of [16]. However, for non-preemptive schedules, our results are tighter and our techniques require less analysis time. The authors of [17] have shown that an SDFG can be used to consider an application with resource sharing possibilities; they perform buffer sizing under a throughput constraint considering a given schedule for the actors using a shared resource. For shared resource analysis, they use event-models [18] which is based on realtime-calculus [19]. Our approach differs from [17], since modeling schedules directly into SDFGs enables us to use dedicated analysis for dataflow graphs. Moreover, the 
technique of [17] can only handle an SDFG with limited types of cycles, such as cycles formed by self-edges or the back edges modeling the buffer capacity between two actors. However, staying in dataflow domain, as is done in our technique, does not impose such a limitation on the graph structure.

Ref [20] uses some new (custom) components, e.g., if then - else, to model schedules in an SDFG. These components are not supported by the common model-based designflows using SDFGs (e.g., [1], [5]-[8]) and cannot be modeled in an SDFG using the basic elements of an SDFG (i.e., actors and channels). Our techniques eliminate the need for any new (custom) component. As a result, any analysis technique for SDFGs is directly applicable to the schedule-extended SDFG.

\section{SYNCHRONOUS DATAFLOW GRAPHS}

Let $\mathbb{N}$ denote the positive natural numbers and $\mathbb{N}_{0}=\mathbb{N} \cup$ $\{0\}$. Consider Ports as a set that contains all ports; each port $p \in$ Ports has a finite rate $\operatorname{Rate}(p) \in \mathbb{N}$. An actor $a_{i}$ is a tuple (In,Out) consisting of a set In $\subseteq$ Ports of input ports and a set $O u t \subseteq$ Ports of output ports with $I n \bigcap$ Out $=\emptyset$.

Definition 1. (SDFG) An SDFG is a tuple $(A, C)$ consisting of a finite set $A$ of actors and a finite set $C \subseteq$ Ports $^{2}$ of channels. The channel source is an output port of an actor and the channel destination is an input port of an actor. Each port of an actor is connected to only one channel and each channel end is connected to a single port. For every actor $a_{i}=($ In, Out $) \in A, \operatorname{InC}\left(a_{i}\right)$ represents all channels connected to the ports in In and OutC $\left(a_{i}\right)$ represents all channels connected to the ports in Out.

The SDFG of Figure 1 has four actors $(A=$ $\left.\left\{a_{0}, a_{1}, a_{2}, a_{3}\right\}\right)$ and three channels $\left(C=\left\{c_{0}, c_{1}, c_{2}\right\}\right)$. Actors communicate with tokens sent from one actor to another over the channels. Tokens are depicted with a solid dot (and an attached number in case of multiple tokens). An essential property of SDFGs is that every time an actor fires (executes) it consumes a fixed number of tokens from its input edges and produces a fixed number of tokens on its output edges. These numbers are called the rates (indicated next to the channel ends when the rates are larger than 1). The rates determine how often actors have to fire with respect to each other such that the distribution of tokens over all channels is not changed. This property is captured in the repetition vector [1] of an SDFG. The repetition vector determines the number of times each actor should be fired in order to bring the SDFG back to its initial token distribution. Notation $\gamma(a)$ refers to the repetition vector value of actor $a$. The repetition vector of the

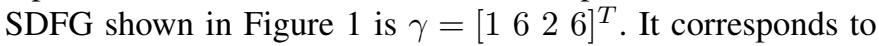
1 firing of $a_{0}, 6$ firings of $a_{1}, 2$ firings of $a_{2}$ and 6 firings of $a_{3}$. Channels can contain different numbers of tokens. A state of an SDFG (represented by $\omega$ ) is described by the number of tokens in all channels of the SDFG. We assume that the initial state of an SDFG is given by an initial token distribution $\omega_{0}$. An actor $a_{i} \in A$ is enabled in an SDFG state $\omega_{j}$ iff $\omega_{j}(c) \geq \operatorname{Rate}(q)$ for each channel $c=(p, q) \in \operatorname{InC}\left(a_{i}\right)$. When an actor $a_{i}$ starts its firing, it removes $\operatorname{Rate}(q)$ tokens from all $(p, q) \in \operatorname{InC}\left(a_{i}\right)$ and when it ends, it produces $\operatorname{Rate}(p)$ tokens on every $(p, q) \in \operatorname{OutC}\left(a_{i}\right)$. Consistency (i.e., the existence of a repetition vector) and absence of deadlock are necessary in practice for SDFGs and can be verified efficiently [21], [22]. Any SDFG which is not consistent requires unbounded memory to execute or deadlocks. Therefore, we limit ourselves to consistent and deadlock-free SDFGs.

\section{SDFG STATIC-ORDER SCHEDULING}

Assume that the initial state $\omega_{0}$ for the SDFG of Figure 1 is equal to $\left(c_{0}, c_{1}, c_{2}\right) \rightarrow(0,0,0)$. Actor $a_{0}$ is enabled in $\omega_{0}$. Firing $a_{0}$ results in a transition from state $\omega_{0}$ to a state $(6,0,0)$. We use this concept of states and transitions to formalize the execution of an SDFG.

Definition 2. (EXECUTION) An execution $\sigma$ of an SDFG is an infinite alternating sequence of states and transitions $\omega_{0} \stackrel{a_{i}}{\longrightarrow}$ $\omega_{1} \stackrel{a_{j}}{\longrightarrow} \cdots$ starting from a designated initial state $\omega_{0}$.

In a multi-processor system, multiple actors may be bound to the same processor. These actors may be enabled at the same time. In such a situation, a schedule is needed to order the firings of the enabled actors on the processor. The fixed port rates make it possible to statically schedule SDFGs with a finite schedule per processor. Such a schedule orders the actor firings on the underlying processor. This type of schedules, which are called periodic static-order schedules (PSOSs), can be repeated indefinitely. A separate PSOS should be constructed for each processor. Each PSOS only includes actors bound to this specific processor. The following definition is used to formally specify a PSOS.

Definition 3. (PERIOdic StATIC-ORder SCHEdULE (PSOS)) A PSOS is a finite ordered list of (a sub-set of) actors in an SDFG $(A, C)$. A PSOS is denoted by $s_{i}=\left\langle\alpha_{1} \alpha_{2} \ldots \alpha_{n}\right\rangle^{*}$ where each $\left.\alpha_{j}\right|_{1 \leq j \leq n}$ is a sub-schedule that represents an actor from $A$ and $n \in \mathbb{N}$ is the length of the schedule $s_{i}$, represented by $n=\left|s_{i}\right|$. The set $A_{i}$ contains all actors that appear at least once in $s_{i}\left(A_{i} \subseteq A\right)$.

A PSOS can be represented in a compact format, called a looped schedule (LS).

Definition 4. (LOOPED SCHEdULE (LS)) A looped schedule, $s_{i}=\left\langle\left(\alpha_{1}\right)^{\beta_{1}}\left(\alpha_{2}\right)^{\beta_{2}} \cdots\left(\alpha_{m}\right)^{\beta_{m}}\right\rangle^{*}$, is defined as a successive execution of $\alpha_{1}$ repeated $\beta_{1}$ times followed by $\alpha_{2}$ repeated $\beta_{2}$ times and so on, where each $\alpha_{j}$ is either an actor firing or a (nested) looped schedule and $\beta_{j} \in \mathbb{N}$.

Definition 5. (Single Appearance Schedule (SAS)) A $L S$ in which each actor appears only once is called a single appearance schedule (SAS).

Assume that the SDFG of Figure 1 is mapped to a platform with two processors $\left(P_{0}\right.$ and $\left.P_{1}\right)$. Actors $a_{0}$ and $a_{2}$ are mapped to $P_{0}$ with the PSOS $s_{0}=\left\langle a_{0}\left(a_{2}\right)^{2}\right\rangle^{*}$ and actors $a_{1}$ and $a_{3}$ are mapped to $P_{1}$ with the PSOS $s_{1}=\left\langle\left(a_{1}\right)^{5}\left(a_{3}\right)^{3} a_{1}\left(a_{3}\right)^{3}\right\rangle^{*}$. $\operatorname{PSOS} s_{0}$ is a SAS while PSOS $s_{1}$ is not. PSOS $s_{1}^{\prime}=$ $\left\langle\left(a_{1}\right)^{3}\left(a_{3}\right)^{3}\right\rangle^{*}$ can be used as a SAS for actors $a_{1}$ and $a_{3}$.

Definition 6. (SDFG ITERATION) Assume $\operatorname{SDFG}(A, C)$ has 
repetition vector $\gamma$. An SDFG iteration is a set of actor firings such that for each $a \in A$, the set contains $\gamma(a)$ firings of a.

Definition 7. (PSOS ITERATION) Let $s_{i}=\left\langle\alpha_{1} \alpha_{2} \ldots \alpha_{n}\right\rangle^{*}$ be a PSOS that schedules actors in $A_{i} \subseteq A$. A PSOS iteration is a sequence of actor firings following the actor order specified in $s_{i}$ starting from actor $\alpha_{1}$ and ending with actor $\alpha_{n}$ with a length equal to $\left|s_{i}\right|$ and including only actors from $A_{i}$.

The actor firing order in an execution $\sigma=\omega_{0} \stackrel{a_{x}}{\longrightarrow}$ $\omega_{1} \stackrel{a_{y}}{\longrightarrow} \cdots$ can be captured using a list $\left\langle a_{x}, a_{y}, \cdots\right\rangle$ where the $j^{\text {th }}$ element in this list is the actor which is fired in the transition from $\omega_{j-1}$ to $\omega_{j}$. The notation $\operatorname{order} \operatorname{List}\left(\sigma, A_{i}\right)$ represents the mentioned list where actors which do not belong to $A_{i}$ are omitted. For example, in the SDFG of Figure 1, consider an execution $\sigma$ that results in list $\quad\left\langle a_{0}, a_{1}, a_{1}, a_{1}, a_{2}, a_{1}, a_{1}, a_{3}, a_{3}, a_{3}, a_{1}, a_{2}, a_{3}, a_{3}, a_{3}\right\rangle$ with $A_{1}=\left\{a_{1}, a_{3}\right\} ;$ then $\operatorname{orderList}\left(\sigma, A_{1}\right)=$ $\left\langle a_{1}, a_{1}, a_{1}, a_{1}, a_{1}, a_{3}, a_{3}, a_{3}, a_{1}, a_{3}, a_{3}, a_{3}\right\rangle$. We say that the corresponding execution of an SDFG satisfies a PSOS when the SDFG is executed according to the PSOS. We use the following to formalize this term.

Definition 8. (SATISFACTION) Let $\sigma$ be an execution of an $\operatorname{SDFG}(A, C)$ and $s_{i}$ a PSOS for actors $A_{i} \subseteq A$. If it exists, let $\sigma^{\prime}$ be the prefix of $\sigma$ such that it contains exactly $\gamma\left(a_{i}\right)$ occurrences of actor $a_{i} \in A_{i}$; $\sigma^{\prime}$ covers $\sigma$ precisely up to the point that one PSOS iteration is executed. Execution $\sigma$ satisfies PSOS $s_{i}$ iff $\sigma^{\prime}$ exists and the ordered list orderList $\left(\sigma^{\prime}, A_{i}\right)$ corresponds to the order specified in $s_{i}$.

When an execution of a consistent and deadlock-free SDFG satisfies the specified PSOSs, the channels of the SDFG need bounded memories (according to Theorem 1 from [23]). The number of actor appearances in the PSOS is a fraction or multiple of its repetition vector entry. Formally, each actor $a_{i}$ in the PSOS should appear $r \cdot \gamma\left(a_{i}\right)$ times in the PSOS (with $r=\frac{u}{v}$ where $u, v \in \mathbb{N}$ ) and the value $r$ is identical for all actors in the PSOS [9]. This follows from the SDFG property that firing each actor as often as indicated in the repetition vector results in a token distribution that is equal to the initial token distribution. In the paper, the term normalized PSOS is used to refer to a PSOS with $r$ equal to 1 .

Definition 9. (NORMALIZED PSOS) A PSOS $s_{i}$ is called normalized iff each actor $a_{j} \in A_{i}$ appears $\gamma\left(a_{j}\right)$ times in one iteration of the PSOS $s_{i}$.

We limit ourselves in the remainder to PSOSs in which $r$ is a unit fraction (i.e., $r=\frac{u}{v}$ with $u=1$ and $v \in \mathbb{N}$ ), although our technique can also be directly applied to model other PSOSs (i.e., in which $u \in \mathbb{N}$ ).

\section{Modeling Periodic Static-Order Schedules}

In this section, we introduce a technique to model PSOSs in an SDFG. We first illustrate all ingredients of our approach through a running example, and then discuss the algorithm and the main steps in the algorithm in more detail. Note that a schedule is correctly modeled if and only if any execution of the schedule-extended graph satisfies the schedule and if any execution of the original graph that satisfies the schedule is still feasible in the schedule-extended graph.

\section{A. Running example}

Here we briefly introduce our approach in modeling a PSOS in an SDFG using a running example. For this purpose consider the example SDFG shown in Figure 1 and the PSOS $s_{1}=\left\langle\left(a_{1}\right)^{5}\left(a_{3}\right)^{3} a_{1}\left(a_{3}\right)^{3}\right\rangle^{*}$ which is a schedule for $a_{1}$ and $a_{3}$. Our approach captures this schedule in the SDFG in three steps, that (i) remove auto-concurrency, (ii) avoid interiteration execution, and (iii) enforce the correct scheduling decisions.

In the example SDFG, a single firing of $a_{0}$ produces 6 tokens in channel $c_{0}$; then 6 firings of $a_{1}$ can be performed simultaneously. This simultaneous firing of an actor is called auto-concurrency; in practice, this corresponds to executing multiple instances of a function (task) in parallel. Autoconcurrency for an actor cannot be handled in a real hardware platform, unless more than one processor is allocated for that actor. In this work, we focus on the case that an actor is mapped to one processor. Hence, auto-concurrency must be removed from the model. To sequentialize firings of an actor, a self-edge with one initial token must be added to that actor; this way auto-concurrency can be removed for that actor. Figure 3(a) shows the example SDFG of Figure 1 in which any auto-concurrency related to $a_{1}$ and $a_{3}$ is removed by adding two self-edges (shown in red).

In one PSOS iteration, each actor must fire a specific number of times. Actors must not be able to get fired more than the number of times indicated by the PSOS. Consider the following situation in the example SDFG of Figure 1. Two firings of $a_{0}$ provide 12 tokens in channel $c_{0}$; this number of tokens is enough for 12 firings of the actor $a_{1}$. The first 6 firings of $a_{1}$ belong to the first iteration and the second 6 firings belong to the second iteration. The second 6 firings of $a_{1}$ can occur before the completion of the first PSOS iteration of $s_{1}$; in this case, the resulting execution does not satisfy the given PSOS $s_{1}$. This situation is called inter-iteration execution. To prevent inter-iteration execution related to $s_{1}$, we create a dependency from the last actor appearing in $s_{1}$ to the first actor appearing in $s_{1}$; see the blue elements in Figure 3(b). This dependency limits the firing of the first actor to a number of firings required in one PSOS iteration.

In the SDFG of Figure 3(b), consider the case that actors $a_{0}, a_{1}$ and $a_{2}$ have fired 1,3 and 1 times, respectively. The initial token distribution is changed to the distribution shown in Figure 3(c). In this graph, both $a_{1}$ and $a_{3}$ from PSOS $s_{1}$ are enabled; but, only the firing of $a_{1}$ must be granted at this point to form an execution that satisfies the given PSOS $s_{1}$. We call such a state in which several actors are enabled a decision state. Later on, a precise definition is given for this concept. According to schedule $s_{1}$, actor $a_{3}$ must get enabled after 5 firings of $a_{1}$. For this purpose, a dependency is created from $a_{1}$ to $a_{3}$ (shown with green actor and channels in Figure 3(d)); this new dependency prevents $a_{3}$ from getting enabled unless $a_{1}$ has completed 5 firings. Another decision state can be found after $a_{1}$ has completed 5 firings; once again, both $a_{1}$ and $a_{3}$ 


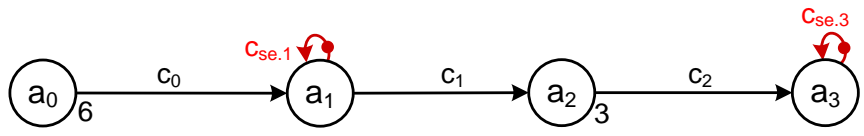

Auto-concurrency

(a) Auto-concurrency has been removed.

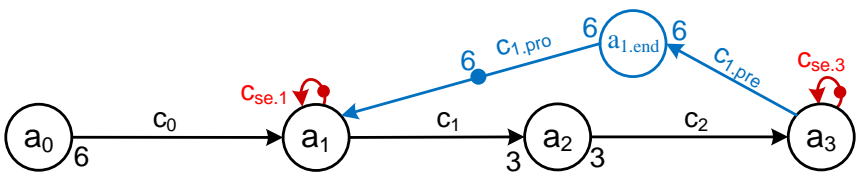

Auto-concurrency

Inter-iteration execution

(b) Auto-concurrency and inter-iteration execution have been removed.

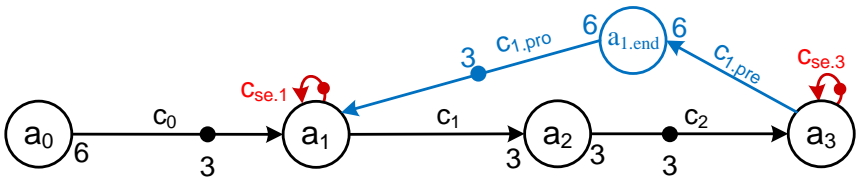

Auto-concurrency

Inter-iteration execution

(c) SDFG of Figure 3(b) after 1, 3 and 1 firings of $a_{0}, a_{1}$ and $a_{2}$ resp., leading to a decision state in which both actors $a_{1}$ and $a_{3}$ are enabled.

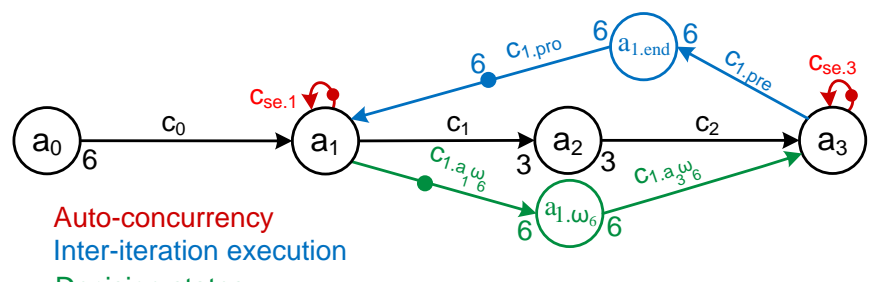

Decision states

(d) Creating a dependency for the first decision state.

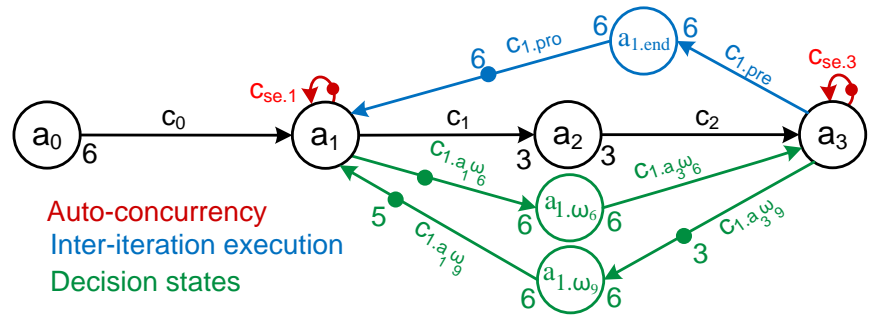

(e) Creating a dependency for the second decision state.

Fig. 3. Step-by-step modeling of the PSOS $s_{1}$ in the SDFG of Figure 1.

from PSOS $s_{1}$ are enabled at this point; but, the firing of $a_{3}$ must take place. For this purpose, a dependency is created from $a_{3}$ to $a_{1}$ (see Figure 3(e)). This new dependency prevents $a_{1}$ from getting enabled from the current state onwards unless $a_{3}$ has completed 3 firings. The 5 initial tokens on the added input channel to $a_{1}$ ensure that the first 5 firings of $a_{1}$ can take place as planned. The SDFG of Figure 3(e) shows the final solution that models the PSOS $s_{1}$ in the SDFG of Figure 1.

\section{B. The algorithm}

Algorithm 1 captures our technique, called decision state modeling (DSM). DSM accepts an SDFG and one or several
PSOSs as its input. In the algorithm $n+1\left(n \in \mathbb{N}_{0}\right)$ is the number of processors (or input PSOSs). DSM ensures that actor firings of each PSOS follow the specified order in that PSOS; the output of DSM is a new SDFG that models the provided PSOSs in the input SDFG. Figure 4 depicts the corresponding SDFG of Figure 1 which models the PSOSs $s_{0}$ and $s_{1}$ using DSM. The remainder of this section discusses the three main steps of the algorithm - removing auto-concurrency, avoiding inter-iteration execution, enforcing correct decisions in decision states - in detail.

The description of some basic functions used in Algorithm 1 is as follows. The function $A A\left(G, a_{n e w}\right)$ is responsible to include the actor $a_{\text {new }}$ in the SDFG $G$. The function $A C\left(G, c_{n e w}, a_{s r c}, a_{d s t}\right.$, srcRate, dstRate, initTok) adds the channel $c_{\text {new }}$ from source actor $a_{s r c}$ to destination actor $a_{d s t}$; the production rate of $a_{s r c}$ on this channel is equal to srcRate and the consumption rate of $a_{d s t}$ on this channel is equal to dstRate; this channel is initialized with initTok initial tokens. The function $C N T\left(a_{j}, s_{i}\right)$ returns the number of times that the actor $a_{j}$ is fired in one iteration of PSOS $s_{i}$. The function $B E F\left(a_{k}, j, s_{i}\right)$ returns the number of times that $a_{k}$ appears from the first position in the PSOS $s_{i}$ to and including the $j^{\text {th }}$ position in the PSOS $s_{i}$; the function $A F T\left(a_{k}, j, s_{i}\right)$ returns the number of times that $a_{k}$ appears from the $(j+1)^{t h}$ position in the PSOS $s_{i}$ to the last position in the PSOS $s_{i}$.

\section{Auto-concurrency}

An actor $a_{i} \in A$ can be enabled multiple times simultaneously in an SDFG state $\omega_{j}$ if $\omega_{j}(c) \geq k \cdot \operatorname{Rate}(q)$ for each channel $c=(p, q) \in \operatorname{InC}\left(a_{i}\right)$ where $k \in \mathbb{N}, k \geq 2$. This is called auto-concurrency. The firings of actor $a_{i}$ should occur sequentially according to the PSOS to which $a_{i}$ belongs. This sequential execution can be enforced by adding a self-edge with one initial token to actor $a_{i}$ (Line 1 in Algorithm 1). In Figure 4, channels $c_{s e .0}-c_{s e .3}$ (shown in red) are used to prevent any auto-concurrency in the SDFG of Figure 1.

\section{Inter-iteration execution}

Consider actor $a_{0}$ in the SDFG of Figure 1; the $1^{\text {st }}$ firing of $a_{0}$ belongs to the $1^{\text {st }}$ PSOS iteration of $s_{0}$ and the $2^{\text {nd }}$ firing of $a_{0}$ belongs to the $2^{\text {nd }}$ PSOS iteration of $s_{0}$ and so on. Since $a_{0}$ has no input channel, it can always be fired regardless of other actors in the graph. This behavior, which is called inter-iteration execution, can prevent an SDFG execution from satisfying the given PSOSs. Inter-iteration execution happens when one PSOS iteration has not been completed and an actor

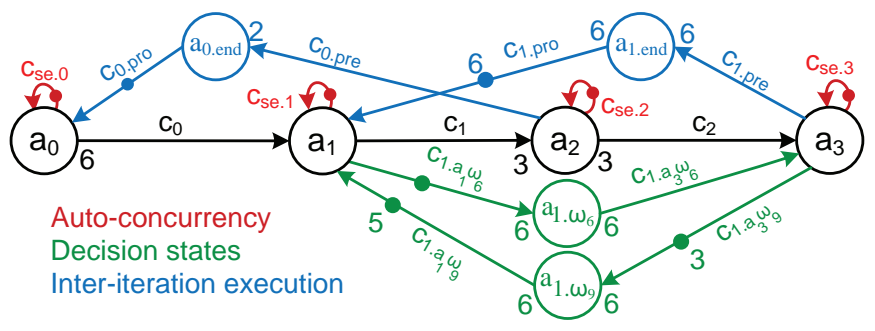

Fig. 4. PSOSs $s_{0}$ and $s_{1}$ modeled in the SDFG of Figure 1 using DSM. 

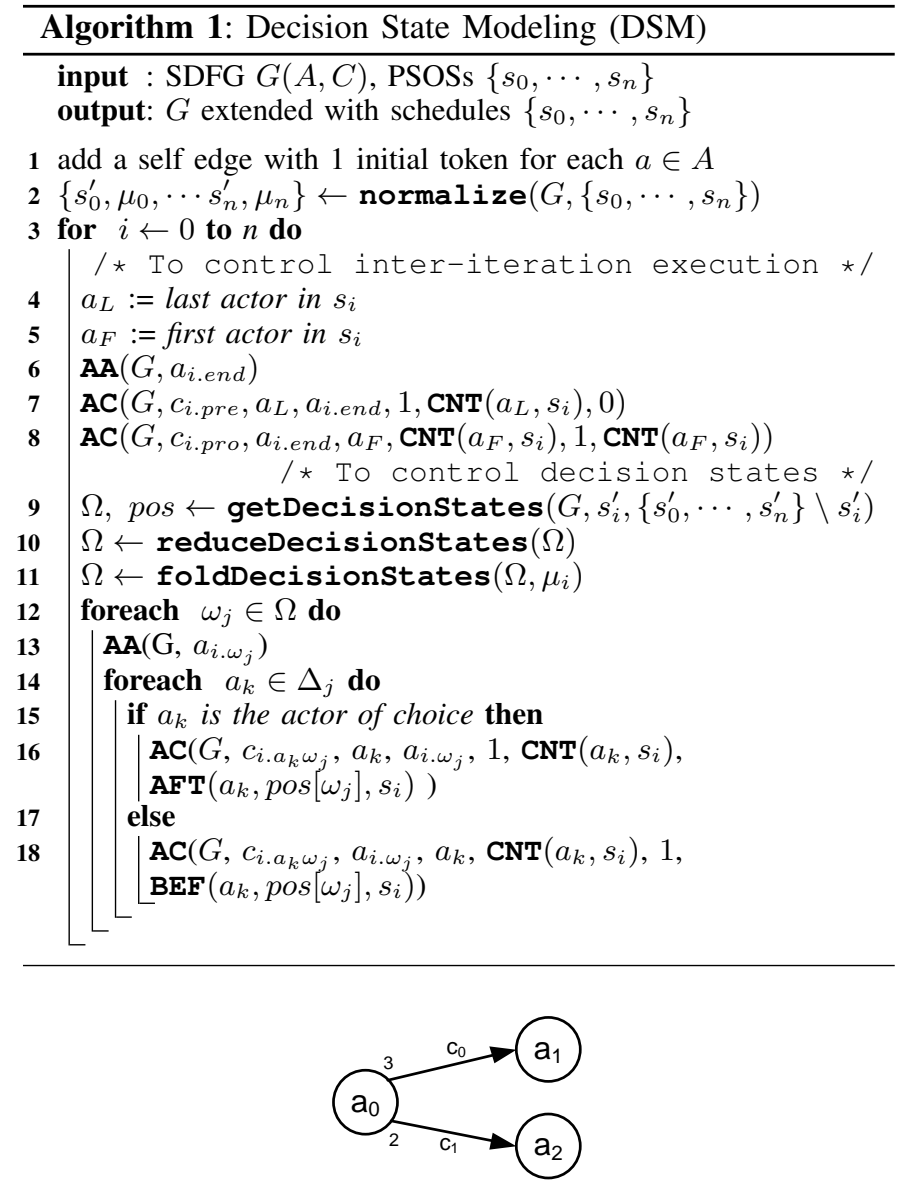

Fig. 5. An example SDFG.

from that PSOS can proceed its firings beyond the current PSOS iteration. Lines 4-8 in Algorithm 1 are used to control this undesirable actor enabling. This part of the algorithm adds (per PSOS) one actor and two channels to create a dependency between the last and first actor appearing in the PSOS. The added components limit, within one PSOS iteration, the firing of the first actor in the PSOS (i.e., $a_{F}$ ) to the count of actor $a_{F}$ (i.e., $\left.C N T\left(a_{F}, s_{i}\right)\right)$ in one iteration of the PSOS $s_{i}$. The next iteration of the PSOS $s_{i}$ can only commence if the last actor in PSOS $s_{i}$ (i.e., $\left.a_{L}\right)$ fires $C N T\left(a_{L}, s_{i}\right)$ times in one iteration of the PSOS $s_{i}$. In other words, the next iteration of a PSOS can only commence after the completion of the current iteration of this PSOS. In Figure 4, actor $a_{0 . e n d}$ and channels $c_{0 . p r e}$ and $c_{0 . p r o}$ are added to prevent any inter-iteration execution in PSOS $s_{0}$. Actor $a_{1 . e n d}$ and channels $c_{1 \text {.pre }}$ and $c_{1 \text {.pro }}$ are added to prevent any inter-iteration execution in schedule $s_{1}$. These elements are shown in our example in blue in Figure 4.

\section{E. Decision states}

This sub-section presents the third step of DSM. It first defines the concept of a decision state and then proceeds with the algorithm for identifying decision states; after explaining two optimization steps, it ends with the technique to enforce the appropriate schedule decisions.

1) Concept: Multiple different actors mapped to a single processor may be enabled in a specific state. Here, we describe

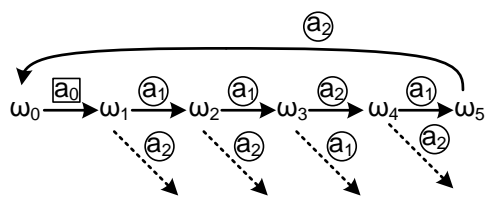

Fig. 6. The state space of the SDFG of Figure 5 when PSOSs $s_{0}^{\prime \prime}=\left\langle a_{0}\right\rangle^{*}$ and $s_{1}^{\prime \prime}=\left\langle\left(a_{1}\right)^{2} a_{2} a_{1} a_{2}\right\rangle^{*}$ are used.

such situations in an SDFG execution. Consider the SDFG in Figure 5. Assume that $a_{0}$ is mapped to processor $P_{0}$ with PSOS $s_{0}^{\prime \prime}=\left\langle a_{0}\right\rangle^{*}$ and $a_{1}$ and $a_{2}$ are mapped to processor $P_{1}$ with PSOS $s_{1}^{\prime \prime}=\left\langle\left(a_{1}\right)^{2} a_{2} a_{1} a_{2}\right\rangle^{*}$. For brevity, we only discuss the actors mapped to processor $P_{1}$. The corresponding state space - for one SDFG iteration - when executing our example SDFG using the PSOSs $s_{0}^{\prime \prime}$ and $s_{1}^{\prime \prime}$ is visualized in Figure 6. In this figure, the actors mapped to processor $P_{0}$ $\left(P_{1}\right)$ are surrounded by a square (circle). Auto-concurrency and inter-iteration execution are excluded using the constructs introduced in Section V-C and Section V-D resp. The periodic behavior of the PSOSs is obvious from the state space where one SDFG iteration moves the graph to its initial state, i.e., $\omega_{0}$ (see Figure 6). There are some states in Figure 6 in which more than one actor is enabled (e.g., $\omega_{1}-\omega_{5}$ ) on processor $P_{1}$. In such a situation, the execution related to those actors can deviate from the specified PSOS. We use the following definition to formalize such a situation.

Definition 10. (DECISION STATE) Consider the PSOS $s_{i}$ as a schedule for actors $A_{i} \subseteq A$ and an execution $\sigma$ of an SDFG $(A, C)$ which satisfies PSOS $s_{i}$. A state $\omega_{j} \in \sigma$ is a decision state iff multiple different actors from $A_{i}$ are enabled in $\omega_{j}$.

We use $\Omega$ to refer to the finite set containing all decision states occurring in the execution of an SDFG up-to one iteration of a PSOS. The following terminology describes the enabled actors in a decision state.

Definition 11. (OPPONENT ACTOR SET) Let $\omega_{j} \in \Omega$ be a decision state for PSOS $s_{i}$. The opponent actor set $\Delta_{j}$ of the decision state $\omega_{j}$ is a finite set which contains all actors that are enabled in decision state $\omega_{j}$ and that belong to $A_{i}$.

The finite set $\Delta_{j}$ represents the opponent actors in the decision state $\omega_{j} \in \Omega$. One of the enabled actors in a decision state $\omega_{j}$, in line with the given PSOS $s_{i}$, should be selected to fire. The following is used to describe such an actor.

Definition 12. (ACTOR OF CHOICE) Consider the PSOS $s_{i}$ which schedules actors $A_{i} \subseteq A$ and the opponent actor set $\Delta_{j}$ of the decision state $\omega_{j}$ in an execution $\sigma$ of the SDFG $G(A, C)$ which satisfies $s_{i}$. An actor $a_{c} \in \Delta_{j}$ is called the actor of choice of the decision state $\omega_{j}$ iff the firing of actor $a_{c}$ in state $\omega_{j}$ is a necessity for the execution $\sigma$ in order to satisfy the PSOS $s_{i}$. Since a PSOS specifies a fixed firing order, there can only be a single actor of choice in any decision state.

Lines 9-18 in DSM show how we deal with nondeterministic execution due to decision states. DSM models the given PSOSs one-by-one iteratively. The ordering of 

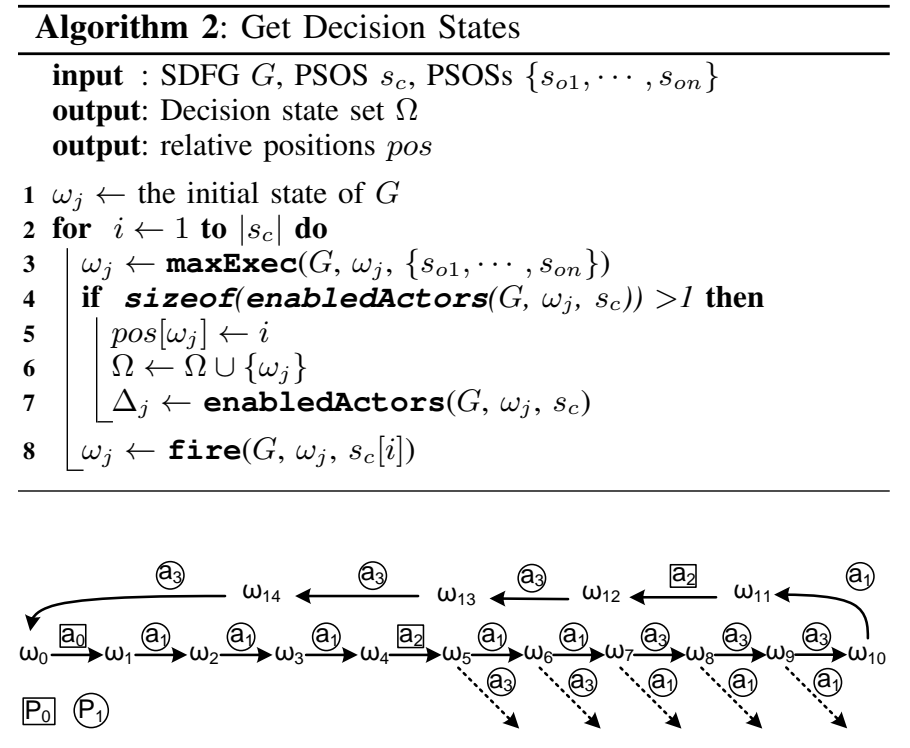

Fig. 7. The state space of the SDFG of Figure 1 when the PSOS $s_{1}=$ $\left\langle\left(a_{1}\right)^{5}\left(a_{3}\right)^{3} a_{1}\left(a_{3}\right)^{3}\right\rangle^{*}$ is the schedule of interest (i.e., $\left.s_{c}\right)$ in Algorithm 2.

PSOSs in DSM does not have any impact on the final behavior. In each iteration of the for-loop in line 3 , we enforce the execution of the actors in the current schedule of interest (i.e., schedule $s_{i}$ ) to follow schedule $s_{i}$. The next sub-section explains how decision states of the schedule of interest are extracted. At the same time, a value is preserved for each decision state that captures the relative position of that decision state with respect to the beginning of the schedule of interest; the notation $\operatorname{pos}\left[\omega_{j}\right]$ refers to this position for $\omega_{j}$. For example, in the SDFG of Figure 1, pos $\left[\omega_{6}\right]=5$ since the relative position of $\omega_{6}$ with respect to the beginning of the schedule of interest (i.e., $s_{1}$ ) is 5 (in Figure 7 consider 4 firings related to $s_{1}$ have been occurred before $\omega_{6}$ and the $5^{\text {th }}$ actor firing related to $s_{1}$ is going to happen in $\left.\omega_{6}\right)$. For each $\omega_{j} \in \Omega$ extracted for $s_{i}$, DSM adds an actor $\left(a_{i . \omega_{j}}\right.$ in line 13) and one channel between the new actor $a_{i . \omega_{j}}$ and each opponent actor in the set $\Delta_{j}$ (lines 14-18 in Algorithm 1). The elements added in each decision state (e.g., $\omega_{j}$ ) postpone the execution of the actors in $\Delta_{j} \backslash\left\{a_{c}\right\}$ to the state after decision state $\omega_{j}$. Hence, $a_{c}$ (i.e., the actor of choice) is the only actor which can be fired in the state $\omega_{j}$.

2) Decision state identification: Algorithm 2 shows our technique to detect all decision states within PSOS $s_{c}$. Assume $s_{c}$ is a PSOS for the actors mapped to processor $P_{c}$. Schedules $s_{o 1} \cdots s_{o n}$ are PSOSs for the other actors of the SDFG mapped to the other processors (denoted by $P_{o 1} \cdots P_{o n}$ ). The output of Algorithm 2 is a set that contains all decision states for PSOS $s_{c}$. This algorithm also returns the relative positions of decision states with respect to the beginning of $s_{c}$. In Algorithm 2, the input schedules are normalized PSOSs. The function normalize (in line 2 of Algorithm 1) normalizes the input PSOSs. The function returns the normalized PSOSs along with their normalization factors. The normalized PSOS $s_{x}^{\prime}$ can be achieved by repeating $\mu_{x}$ times the input PSOS $s_{x}$ (i.e., $\left.s_{x}^{\prime}=\left(s_{x}\right)^{\mu_{x}}\right) . \mu_{x}$ is the normalization factor of $s_{x}$ and can be calculated by dividing the repetition vector entry of an arbitrary actor in $s_{x}$ by the count of that actor in the PSOS $s_{x}$ (in our running example, $\mu_{0}$ and $\mu_{1}$ are 1 ).

After normalizing the input schedules, all situations that can lead to multiple actors (mapped to the same processor) being ready to fire must be discovered. An actor in the schedule of interest $s_{c}$ could be affected by the execution of an actor in the other schedules as well as by another actor in $s_{c}$. Processors can run at different clock rates; these differences and interprocessor dependencies cause variation in the number of tokens on the inter-processor channels originating from the actors mapped to the other processors to the actors mapped to the processor of interest (i.e., $P_{c}$ ). The number of tokens on the input channels of an actor determines whether an actor is enabled or not. To determine any possible actor enabling within $s_{c}$, a necessary and sufficient number of tokens on all inter-processor channels entering to the actors mapped to processor $P_{c}$ must be considered. We will now explain what necessary and sufficient number of tokens means in our algorithm. Each iteration of the schedule of interest $s_{c}$ requires that the actors mapped to the other processors are fired upto at most their repetition vector entry values. Hence, only executing one iteration of the other schedules $s_{o 1} \cdots s_{o n}$ is enough to provide a necessary number of tokens on interprocessor channels entering to the actors mapped to processor $P_{c}$. More than one iteration for the other schedules $s_{o 1} \cdots s_{o n}$ may be feasible; this may cause an actor in $s_{c}$ to be enabled more than its count in one iteration of $s_{c}$. The inter-iteration prevention constructs introduced in Section V-D control this undesired actor enabling. So, we only extract decision states within one iteration of the normalized schedule to provide a sufficient number of tokens.

Also, DSM does not impose any limitation between PSOSs since no dependency is created between actors mapped to different processors. PSOSs can independently be iterated if the dependencies in the SDFG allow that. We allow the actors on the other processors to be executed (according to their schedules) as much as they can; the corresponding execution is named maximal execution. The maximal execution will stop at one point either due to a dependency on the actors on the processor $P_{c}$ or because one PSOS iteration is completed. The SDFG state (denoted by $\omega_{j}$ in Algorithm 2) should be kept during the operation of the algorithm. The maximal execution is represented by the function maxExec in Algorithm 2. After one maximal execution, the number of tokens on the interprocessor channels entering into the actors on the processor $P_{c}$ determines any possible enabled actor. The preserved state (i.e., $\omega_{j}$ ) will be added to the decision state set $(\Omega)$ if more than one actor on the processor $P_{c}$ is enabled at this state (line 6 in Algorithm 2). The current position (i.e., $i$ ) in the schedule of interest $s_{c}$ is also preserved for the discovered decision state (see line 5 in Algorithm 2). All enabled actors will be recorded as opponent actors of the state $\omega_{j}$ (line 7 in Algorithm 2). The execution of the actors on the processor $P_{c}$ is continued by executing the enabled actor in line with $s_{c}$ to determine all possible decision states (line 8 in Algorithm $2)$. The function $\operatorname{fire}\left(G, \omega_{j}, s_{c}[i]\right)$ fires the actor at the $i^{t h}$ position in the PSOS $s_{c}$. The process is repeated until a full iteration of the PSOS has been examined. In the end, the set 


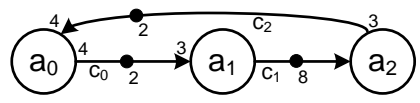

Fig. 8. A third example SDFG.

$\Omega$ contains all possible decision states when executing $s_{c}$.

Figure 7 depicts the resulting state space from applying Algorithm 2 on the SDFG of Figure 1 when $s_{1}$ is the schedule of interest (i.e., $s_{c}$ ). In the SDFG of Figure 1, five consecutive decision states $\left(\Omega=\left\{\omega_{5} \cdots \omega_{9}\right\}\right)$ have been found for $s_{1}$ and no decision state has been found for $s_{0}$ (see Figure 7).

3) Redundant decision states: Here, we explain an optimization proposed in DSM to remove unnecessary decision states. DSM adds some components (per decision state) to create a dependency from the actor of choice of a decision state to the other opponent actors of that decision state. Such a dependency delays the firing of those opponent actors to the state after the decision state. The added components are explained in detail in Section V-E5.

It is possible to have several consecutive decision states which are delaying the firing of an actor to several states later. For example, three consecutive decision states $\left(\omega_{7}-\omega_{9}\right)$ exist in Figure 7 that all delay the firing of $a_{1}$; the added components in $\omega_{7}$ delay the sixth firing of $a_{1}$ to $\omega_{8}$; the added components in $\omega_{8}$ delay the sixth firing of $a_{1}$ to $\omega_{9}$; and so on. The latest decision state in the sequence of decision states $\omega_{7}-\omega_{9}$ is enough to delay the firing of $a_{1}$ to $\omega_{10}$. Hence, the decision states $\omega_{7}-\omega_{8}$ are redundant and can be removed from the decision state set $\Omega$. The function reduceDecisionStates is responsible for removing redundant decision states. Note that it would be possible to perform this reduction during the decision state identification step. This optimization can significantly reduce the number of extra components in the final SDFG. Decision state $\omega_{5}$ is also redundant according to our optimization. So, only two decision states $\omega_{6}$ and $\omega_{9}$ are necessary to model $s_{1}$ in the SDFG of Figure 1.

4) Decision state folding: In Algorithm 1, the input PSOSs are normalized to find all decision states. The normalization of PSOSs is required to explore sufficient states of an SDFG. Consider PSOSs $s_{2}=\left\langle a_{0}\right\rangle^{*}$ and $s_{3}=\left\langle a_{2} a_{1}\right\rangle^{*}$ for our second example SDFG in Figure 8. To obtain normalized PSOSs, $\mu_{2}$ and $\mu_{3}$ must be equal to 3 and 4 respectively. This leads to the following normalized PSOSs: $s_{2}^{\prime}=\left\langle\left(a_{0}\right)^{3}\right\rangle^{*}$ and $s_{3}^{\prime}=\left\langle\left(a_{2} a_{1}\right)^{4}\right\rangle^{*}$. Decision state identification for $s_{3}^{\prime}$ results in 5 decision states. $\underbrace{\left(\begin{array}{c}a_{2} \\ -\end{array}\right)\left(\begin{array}{c}a_{1} \\ a_{2}\end{array}\right)}_{1^{\text {st }}} \underbrace{\left(\begin{array}{c}a_{2} \\ a_{1}\end{array}\right)\left(\begin{array}{c}a_{1} \\ a_{2}\end{array}\right)}_{3^{\text {rd }}} \underbrace{\left(\begin{array}{c}a_{2} \\ a_{1}\end{array}\right)\left(\begin{array}{c}a_{1} \\ a_{2}\end{array}\right)}_{5^{\text {th }}} \underbrace{\left(\begin{array}{c}a_{2} \\ -\end{array}\right)\left(\begin{array}{c}a_{1} \\ -\end{array}\right)}_{7^{\text {th }}}$ shows the corresponding execution of $s_{3}^{\prime}$. In construct $\left(\begin{array}{l}a_{x} \\ a_{y}\end{array}\right), a_{x}$ is the enabled actor in line with the PSOS and $a_{y}$ is the other enabled actor if any at all. In this execution, the $1^{s t}, 3^{r d}, 5^{\text {th }}$ and $7^{\text {th }}$ states are similar in behavior since the same actor (i.e., $a_{2}$ ) is expected to fire in all of those states.

Modeling a repetitive behavior for a PSOS $s_{i}$, also models this behavior for its normalized PSOS (i.e., $s_{i}^{\prime}=\left(s_{i}\right)^{\mu_{i}}$ ). Using this property, we can merge decision states appearing in all $\mu_{i}$ repetitions of the PSOS $s_{i}$. We call this optimization decision state folding (line 11 in Algorithm 1). Folding groups the similar states. In our example, the $1^{s t}, 3^{r d}, 5^{t h}$ and $7^{t h}$ states are grouped and represented with one state. Similarly, the $2^{\text {nd }}$, $4^{\text {th }}, 6^{\text {th }}$ and $8^{\text {th }}$ states are grouped. So, the above execution shrinks to $\left(\begin{array}{l}a_{2} \\ a_{1}\end{array}\right)\left(\begin{array}{l}a_{1} \\ a_{2}\end{array}\right)$. After grouping all similar states in the original execution into a representative state, it is considered a decision state if any of the group members is a decision state. In practice, a decision state in a state of the new folded execution will be considered as a decision state for each of the equivalent states in the original execution. This cannot violate the execution according to the input PSOS because DSM ensures that only the actor of choice executes in a decision state. This optimization could reduce the number of decision states up to $\mu_{i}$ times in a normalized PSOS $s_{i}^{\prime}$. The firings related to those actors enabled in the last state except the actor of choice of that state are supposed to happen in subsequent PSOS iterations; this is already ensured by preventing interiteration execution (see Section V-D). Hence, after folding, the last state can be ignored as a decision state. In our third example, decision state folding reduces the number of decision states from 5 to 1 for the PSOS $s_{3}$.

5) Enforcing a schedule in decision states: In our first example SDFG, only two actors are enabled in decision state $\omega_{6}$ (i.e., $\Delta_{6}=\left\{a_{1}, a_{3}\right\}$ ) (see Figure 7). Actor $a_{1}$ is the actor of choice in $\omega_{6}$ and $a_{3}$ is the only opponent actor whose execution should be delayed to the state after $\omega_{6}$. To enforce firing of $a_{1}$ and to prevent firing of $a_{3}$ in $\omega_{6}$, DSM creates a dependency from $a_{1}$ to $a_{3}$ by adding actor $a_{1 . \omega_{6}}$ and channels $c_{1 . a_{1} \omega_{6}}$ and $c_{1 . a_{3} \omega_{6}}$. The rates and initial tokens related to the new elements are set in such a way that the execution of the graph in other states are not affected. The actor $a_{1 . \omega_{6}}$ is only responsible for decision state $\omega_{6}$. So, $a_{1 . \omega_{6}}$ needs to only fire once in an iteration. For this purpose, the port rates of $a_{1 . \omega_{6}}$ on its channels (i.e., $c_{1 . a_{1} \omega_{6}}$ and $c_{1 . a_{3} \omega_{6}}$ ) are set to 6 . The added dependency channels from the newly added actor in decision state $\omega_{j}$ (e.g., $a_{1 . \omega_{6}}$ in $\omega_{6}$ ) to the opponent actors which are not the actor of choice (e.g., $a_{3}$ in $\omega_{6}$ ) only provide enough tokens for their execution in states $\omega_{0}-\omega_{j-1}$ (e.g., 0 tokens for $a_{3}$ in $\omega_{0}-\omega_{5}$ ); these actors cannot be enabled due to the lack of initial tokens in the newly added channels in the corresponding decision state (e.g., there will be no token in $c_{1 . a_{3} \omega_{6}}$ in $\omega_{6}$ ). Hence, only the actor of choice amongst the opponent actors of a decision state will be enabled in that state (e.g., only $a_{1}$ can fire in $\omega_{6}$ ). The delayed actors in a decision state (e.g., $\omega_{j}$ ) will have sufficient tokens on the incoming channel from the newly added actor for that decision state (i.e., $a_{i . \omega_{j}}$ ) after firing of the actor of choice in $\omega_{j}$. For example, there will be 6 tokens in channel $c_{1 . a_{1} \omega_{6}}$ after the firing of $a_{1}$ (i.e., the actor of choice) in decision state $\omega_{6}$; hence, the actor $a_{1 . \omega_{6}}$ immediately fires and then provides sufficient tokens for later firings of $a_{3}$. So, the delayed actor in decision state $\omega_{6}$ (i.e., $a_{3}$ ) will no longer be blocked due to the absence of tokens in channel $c_{1 . a_{3} \omega_{6}}$ after the decision state $\omega_{6}$. The firing of actor $a_{1}$ after decision state $\omega_{6}$ produces 1 token in channel $c_{1 . a_{1} \omega_{6}}$ and the firings of actor $a_{3}$ after decision state $\omega_{6}$ consumes 6 tokens from channel $c_{1 . a_{3} \omega_{6}}$; as a result, the number of tokens in the new channels are reset to the initial values at the end of one iteration of the schedule $s_{1}$. Hence, the periodic behavior is also achievable for the added components.

DSM also adds actor $a_{1 . \omega_{9}}$ and channels $c_{1 . a_{1} \omega_{9}}$ and $c_{1 . a_{3} \omega_{9}}$ 


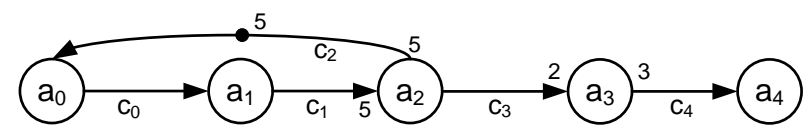

Fig. 9. An example SDFG.

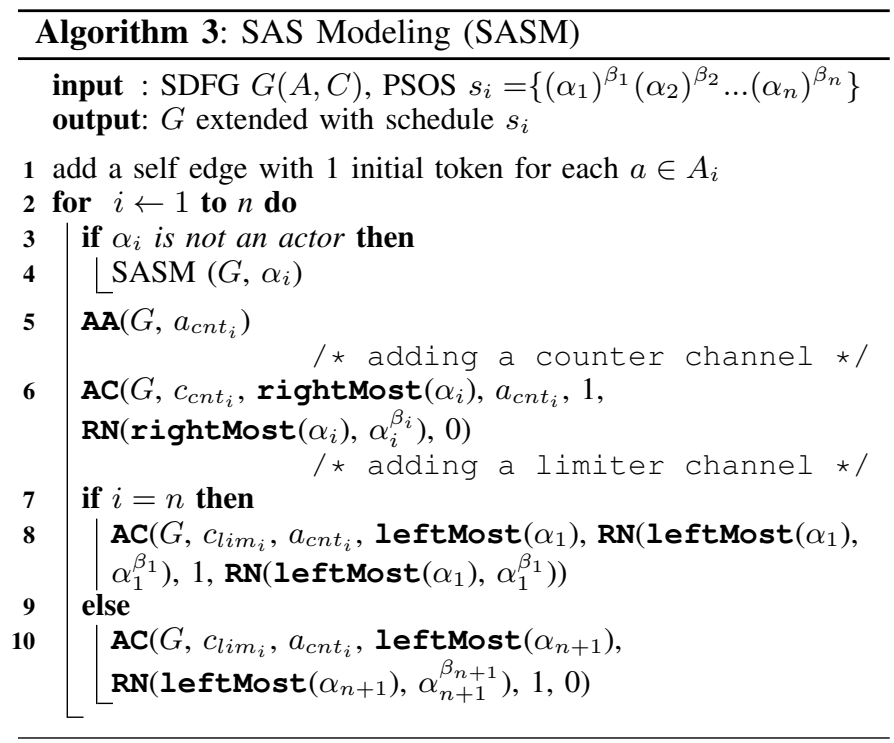

to the graph for the other decision state $\omega_{9}$. The components added in decision state $\omega_{9}$ show similar behavior as the components added in $\omega_{6}$.

\section{Modeling Single Appearance Schedules}

A well-known class of scheduling techniques are single appearance schedules (SASs) in which each actor appears exactly once in the LS form. This aspect makes SASs suitable to minimize code memory size. $s_{2}=\left\langle\left(a_{0} a_{1}\right)^{5} a_{2}\right\rangle^{*}$ is a PSOS and SAS for part of the SDFG (i.e., actors $\left.a_{0}-a_{2}\right)$ in Figure 9.

DSM is able to model any PSOS. However, more compact graphs are possible for SASs. Algorithm 3 presents our single appearance schedule modeling (SASM) technique. Similar to DSM, SASM adds some extra actors and channels to the original SDFG to model the given schedules. The original channels and actors in the schedule-extended SDFG are preserved and distinguishable from the newly added elements by any of our techniques. Hence, both our techniques preserve the original structure of an SDFG. This property can be beneficial when a resource allocation algorithm needs to be applied on the schedule-extended graph; a resource allocation algorithm can easily distinguish an original actor (or channel) from an actor (or channel) which is added to model the schedules.

We know that each actor appears only once in a SAS; this property can help us to model a SAS in an SDFG in a smarter way than DSM does. An actor (or a nested inner LS) should be executed a specific number of times before another actor (or another nested inner LS) starts executing. In $s_{2}=\left\langle\left(a_{0} a_{1}\right)^{5} a_{2}\right\rangle^{*}$, the nested inner LS $\left(a_{0} a_{1}\right)$ must be executed 5 times before $a_{2}$ starts firing. This type of execution control can be handled using a counter construct. The key idea of SASM is to implement a counter concept in the graph.
Later, we explain how we implement such counters to model SASs in an SDFG. Similar to DSM, auto-concurrency can be eliminated by adding a self-edge with 1 initial token to each actor in the SDFG (see line 1 in Algorithm 3). The rest of Algorithm 3 deals with implementing the counter concept.

Figure 10 shows the graph of the SDFG in Figure 9 which models the schedule $s_{2}$ using SASM. Schedule $s_{2}$ has a nested $a_{0} a_{1}$; this can be replaced with $\alpha_{01}$ to form a looped schedule representation (i.e. $s_{2}=\left\langle\left(\alpha_{01}\right)^{5} a_{2}\right\rangle^{*}$ where $\left.\alpha_{01}=a_{0} a_{1}\right)$. A counter in SASM is implemented by one actor $a_{c n t_{i}}$ (e.g., $a_{\text {cnt }_{3}}$ in Figure 10), a counter channel $c_{c t_{i}}$ (e.g., $c_{c n t_{3}}$ ) and a limiter channel $c_{\lim _{i}}$ (e.g., $c_{\lim _{3}}$ ). A counter in SASM has two properties: first, it counts the number of times that element $\alpha_{i}$ (e.g., $\alpha_{01}$ above) is being executed; second, it limits the element $\alpha_{i+1}$ (e.g., $a_{2}$ ) to be executed to $\beta_{i+1}$ times (e.g., 1 as the number of repetitions of $a_{2}$ is one). The counter channel $c_{c n t_{i}}$ (e.g. $c_{c n t_{3}}$ ) is placed from the rightmost actor in $\alpha_{i}$ (e.g., actor $a_{1}$ in $\alpha_{01}$ ) to the actor $a_{c n t_{i}}$ (e.g., $a_{c n t_{3}}$ ); the production rate of the rightmost actor in $\alpha_{i}$ on $c_{c n t_{i}}$ is set to 1 and the consumption rate of $a_{c n t_{i}}$ on $c_{c n t_{i}}$ is set to the number of times that the rightmost actor in $\alpha_{i}$ can be executed in $\alpha_{i}{ }^{\beta_{i}}$ (e.g., 5 as the number of times that $a_{1}$ can be executed in $\alpha_{01}{ }^{5}$ is five). In Algorithm 3, the function rightMost $\left(\alpha_{i}\right)$ (leftMost $\left.\left(\alpha_{i}\right)\right)$ returns the rightmost (leftmost) actor in element $\alpha_{i}$ (e.g. rightMost $\left(\left(a_{0} a_{1}\right)^{5}\right)$ returns actor $\left.a_{1}\right)$. The function $R N\left(a, \alpha_{i}{ }^{\beta_{i}}\right)$ retrieves the count of $a$ in element $\alpha_{i}{ }^{\beta_{i}}$ (e.g. $R N\left(a_{0},\left(a_{0} a_{1}\right)^{5}\right)$ returns 5).

The limiter channel $c_{\text {lim }_{i}}$ (e.g., $c_{\text {lim }_{3}}$ ) is placed from $a_{\text {cnt }_{i}}$ (e.g., $a_{c n t_{3}}$ ) to the leftmost actor in the next element, i.e., $\alpha_{i+1}$ (e.g., $a_{2}$ ). SASM performs the placement of the counter constructs in a circular way. In other words, the next element of $\alpha_{j}$ is considered to be $\alpha_{(j+1)} \bmod n$ where $j \in \mathbb{N} \wedge j \leq n$ for a $\operatorname{LS}\left\{\left(\alpha_{1}\right)^{\beta_{1}}\left(\alpha_{2}\right)^{\beta_{2}} \ldots\left(\alpha_{n}\right)^{\beta_{n}}\right\}$. The production rate of $a_{c n t_{i}}$ on $c_{l i m_{i}}$ is set to the number of times that the leftmost actor in $\alpha_{i+1}$ can be executed in $\alpha_{i+1}{ }^{\beta_{i+1}}$ (e.g., 1 as the number of times that $a_{2}$ can be executed in element $a_{2}$ ) and the consumption rate of the leftmost actor in the next element, i.e., $\alpha_{i+1}$, from $c_{l i m_{i}}$ is set to 1 . So, element $\alpha_{i+1}$ depends on actor $a_{\text {cnt }_{i}}$ (because of $c_{\text {lim }_{i}}$ ) and actor $a_{\text {cnt }}$ depends on $\alpha_{i}$ (because of the $c_{c n t_{i}}$ ); the $\beta_{i}$ executions of $\alpha_{i}$ produce enough tokens on the counter channel $c_{c n t_{i}}$ and then actor $a_{c n t_{i}}$ can be fired. The firing of actor $a_{c n t_{i}}$ provides enough tokens on limiter channel $c_{l i m_{i}}$ to only allow $\beta_{i+1}$ executions for the next element $\alpha_{i+1}$. Hence, by adding these components we enforce that $\alpha_{i+1}$ can be executed $\beta_{i+1}$ times after $\alpha_{i}$ is executed $\beta_{i}$ times.

The limiter channel of the counter construct added for the last element (i.e., $\left(\alpha_{n}\right)^{\beta_{n}}$ ) in a $\operatorname{LS}\left\{\left(\alpha_{1}\right)^{\beta_{1}}\left(\alpha_{2}\right)^{\beta_{2}} \ldots\left(\alpha_{n}\right)^{\beta_{n}}\right\}$ is initialized with some initial tokens to prevent a deadlock in the graph. The number of initial tokens on the limiter channel is set to the count of the left most actor of $\alpha_{1}$ in the first element of that LS (i.e., $\left.\left(\alpha_{1}\right)^{\beta_{1}}\right)$. Line 8 in SASM performs the token initialization. Inter-iteration execution cannot happen because SASM always creates a dependency from the last actor in the schedule to the first actor in the schedule.

In Figure 10, the actor $a_{c n t_{3}}$ is added to count the number of times that the sequence $a_{0} a_{1}$ is executed. The consumption rate of actor $a_{\mathrm{cnt}_{3}}$ on its input channel is 5; this means that 


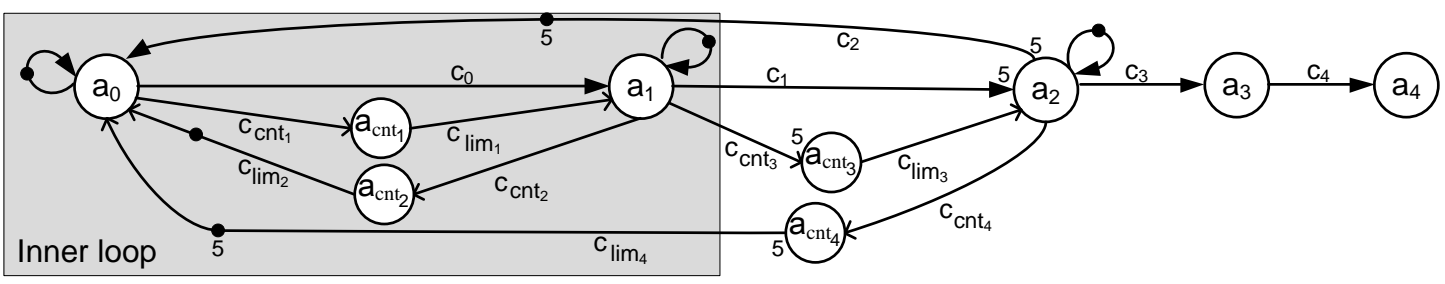

Fig. 10. SDFG of Figure 9 extended with $s_{2}=\left\langle\left(a_{0} a_{1}\right)^{5} a_{2}\right\rangle^{*}$ using SASM.

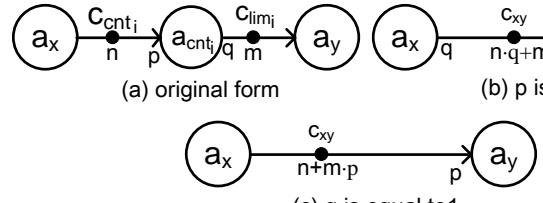

(c) $q$ is equal to 1

Fig. 11. Optimization of SASM.

after 5 executions of sequence $a_{0} a_{1}$ the next actor (i.e., $a_{2}$ ) can be enabled. Also, the actor $a_{c n t_{3}}$ limits the number of times that actor $a_{2}$ should get enabled; this can be done by choosing the value 1 as production rate of actor $a_{c n t_{3}}$ on its output channel. In other words, the actor $a_{2}$ can only fire once because of the limitation imposed by actor $a_{c n t_{3}}$. The actor $a_{c n t_{1}}\left(a_{c n t_{2}}\right)$ is added to ensure the single execution of actors $a_{1}\left(a_{0}\right)$ after the single execution of actor $a_{0}\left(a_{1}\right)$. The actor $a_{c n t_{4}}$ is added to ensure that the sequence $a_{0} a_{1}$ can be executed 5 times after the actor $a_{2}$ is executed once.

SASM is applied recursively (line 4 in SASM) to model the nested LS $\alpha_{i}$. For example, $\operatorname{SASM}\left(G, a_{0} a_{1}\right)$ will be called inside $\operatorname{SASM}\left(G,\left(a_{0} a_{1}\right)^{5} a_{2}\right)$; the result of the recursive call is shown in Figure 10 with a rectangle marking the inner-loop.

Some of the elements added by SASM can be removed without affecting the outcome. Consider Figure 11(a) which contains a counter actor and two channels that can be discarded in the following cases:

- The counter actor $a_{c n t_{i}}$ can be removed if rate $p$ is equal to 1 . The counter actor and two channels in the original form are replaced with channel $c_{x y}$ (see Figure 11(b)).

- The counter actor $a_{c n t_{i}}$ can be removed if rate $q$ is equal to 1 . The counter actor and two channels in the original form are replaced with channel $c_{x y}$ (see Figure 11(c)).

The newly replaced channel $c_{x y}$ is only necessary if there is no other equivalent channel in the original SDFG. The channels $(p, q)$ and $\left(p^{\prime}, q^{\prime}\right)$ which have the same source actor $a_{x} \in A$ and sink actor $a_{y} \in A$ are equivalent if the equation $\frac{\operatorname{Rate}(p)}{\operatorname{Rate}\left(p^{\prime}\right)}=$ $\frac{\operatorname{Rate}(q)}{\operatorname{Rate}\left(q^{\prime}\right)}=\frac{\omega_{0}(c)}{\omega_{0}\left(c^{\prime}\right)}$ is true. Applying these optimizations on Figure 10 replaces all components added by SASM by channel $c_{01}$ (see Figure 12). The SDFG which models schedule $s_{2}$ in the SDFG of Figure 9 with DSM and the HSDFG-based techniques result in a graph with 10 (26) and 13 (21) actors (channels) resp. The SDFG which models the same schedule with SASM only has 5 (9) actors (channels).

\section{Correctness of the Proposed Techniques}

This section presents the theorems related to the correctness of our proposed techniques. A schedule is encoded correctly

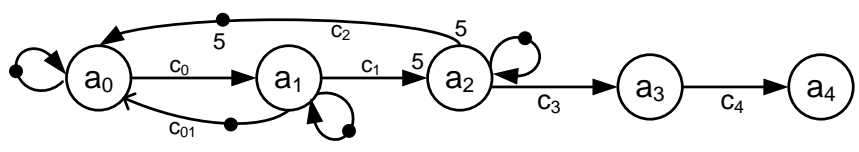

Fig. 12. SDFG of Figure 9 extended with $s_{2}=\left\langle\left(a_{0} a_{1}\right)^{5} a_{2}\right\rangle^{*}$ using optimized SASM.

if any execution of a schedule-extended graph satisfies the encoded schedule and if any execution of the original graph that satisfies the given schedule is still possible in the scheduleextended graph. The proofs of the theorems are given in the appendices of this report.

Assume that the actors and channels added by DSM (or SASM) to model schedule $s_{i}$ in SDFG $G(A, C)$ are denoted by $A_{s_{i}}$ and $C_{s_{i}}$, resp. $G^{\prime}\left(A^{\prime}, C^{\prime}\right)$ is the SDFG that models $s_{i}$ in $G$ using DSM (or SASM) where $A^{\prime}=A \cup A_{s_{i}}$ and $C^{\prime}=C \cup C_{s_{i}}$.

Theorem 1 and Theorem 2 state the correctness of DSM in modeling a single PSOS for a sub-set of the actors of the SDFG. Applying the theorems once for each schedule to be encoded shows the correctness of Algorithm 1.

Note that actors and channels added to model a given schedule do not affect actors that are not part of the schedule. Theorem 1 shows that any execution of the schedule-extended graph satisfies the modeled schedule. Firings of the added actors need to be ignored, which is achieved by the stated condition on the ordered lists resulting from the executions in the schedule-extended and original graphs.

Theorem 1. Consider PSOS $s_{i}$ as a schedule for actors $A_{i} \subseteq$ A from $\operatorname{SDFG} G(A, C)$. Assume $G^{\prime}\left(A^{\prime}, C^{\prime}\right)$ is the $S D F G$ that models $s_{i}$ in $G$ using DSM. For any execution $\sigma^{\prime}$ of $G^{\prime}\left(A^{\prime}, C^{\prime}\right)$ it holds that $\sigma$ satisfies $s_{i}$ where it is assumed that $\sigma$ is the execution of $G(A, C)$ with orderList $(\sigma, A)=$ $\operatorname{order} \operatorname{List}\left(\sigma^{\prime}, A\right)$.

Theorem 2 shows that no execution of the original graph is unnecessarily excluded in the schedule-extended graph. In other words, any execution of the original graph that satisfies a given schedule is still feasible in the schedule-extended graph.

Theorem 2. Consider PSOS $s_{i}$ as a schedule for actors $A_{i} \subseteq$ A from $\operatorname{SDFG} G(A, C)$. Assume $G^{\prime}\left(A^{\prime}, C^{\prime}\right)$ is the $S D F G$ that models $s_{i}$ in $G$ using DSM. For any execution $\sigma$ of $G$ that satisfies $s_{i}$ it holds that there is exactly one $\sigma^{\prime}$ that is an execution of $G^{\prime}\left(A^{\prime}, C^{\prime}\right)$ such that orderList $(\sigma, A)=$ $\operatorname{order} \operatorname{List}\left(\sigma^{\prime}, A\right)$.

The following two theorems state the correctness of SASM. 
They are very similar to the two theorems for DSM.

Theorem 3. Consider SAS $s_{i}=\left\{\left(\alpha_{1}\right)^{\beta_{1}}\left(\alpha_{2}\right)^{\beta_{2}} \ldots\left(\alpha_{n}\right)^{\beta_{n}}\right\}$ as a schedule for actors $A_{i} \subseteq A$ from $\operatorname{SDFG} G(A, C)$. Assume $G^{\prime}\left(A^{\prime}, C^{\prime}\right)$ is the SDFG that models $s_{i}$ in $G$ using SASM. For any execution $\sigma^{\prime}$ of $G^{\prime}\left(A^{\prime}, C^{\prime}\right)$ it holds that $\sigma$ satisfies $s_{i}$ where it is assumed that $\sigma$ is the execution of $G(A, C)$ with $\operatorname{orderList}(\sigma, A)=\operatorname{orderList}\left(\sigma^{\prime}, A\right)$.

Theorem 4. Consider PSOS $s_{i}$ as a schedule for actors $A_{i} \subseteq$ A from SDFG $G(A, C)$. Assume $G^{\prime}\left(A^{\prime}, C^{\prime}\right)$ is the $S D F G$ that models $s_{i}$ in $G$ using DSM. For any execution $\sigma$ of $G$ that satisfies $s_{i}$ it holds that there is exactly one $\sigma^{\prime}$ that is an execution of $G^{\prime}\left(A^{\prime}, C^{\prime}\right)$ such that orderList $(\sigma, A)=$ orderList $\left(\sigma^{\prime}, A\right)$.

\section{EXPERIMENTAL RESULTS}

In this section, we evaluate our techniques experimentally. We first explain the experimental setup. We then evaluate our techniques in terms of the sizes of the schedule-extended graphs, comparing our techniques to that of [12]. We further consider the throughput analysis time when analyzing the schedule-extended graphs obtained by different techniques. Note that the throughput that is achievable for a given schedule is independent of the way it is encoded. It is the analysis time itself that is of interest. Finally, we look at the accuracy of buffer sizing analysis. The accuracy of obtained buffer requirements does depend on the way schedules are encoded.

\section{A. Experimental setup}

The DSM and SASM techniques have been integrated in the $\mathrm{SDF}^{3}$ [28] dataflow tool set, available at http://www.es.ele.tue.nl/sdf3. We use a set of DSP and multimedia applications (see the first column of Table I) to assess our DSM and SASM techniques.

In our experiments, applications are bound to a multiprocessor platform using the technique of [6]. A PSOS determines the actor firing order and as such it influences the enabled actors in a state; as a result, the number of decision states can be different for different PSOSs. The size of the schedule-extended graph using DSM depends on the number of decision states in the given schedules. We use a list scheduling approach from [29] to determine PSOSs for the applications. We use two different variations to verify DSM in different situations. The first list schedule uses forward priorities (Lfp) and the second one uses reverse priorities (Lrp). Actors closer to the inputs of the graph have higher priority in the Lfp schedules compared to actors closer to the outputs of the graph and vice-versa in Lrp schedules.

The scheduling technique presented in [30] is used to derive SASs for our benchmark applications. The technique in [30] also minimizes the required buffer sizes when determining a SAS. However, the technique in [30] cannot directly be used for multi-processors. We have utilized the technique of [30] to find SASs for a multi-processor platform. Initially the binding technique from [6] is used to bind the SDFG to a multi-processor platform. Then, the technique of [30] is applied to the SDFG to derive a SAS for all actors in the SDFG. This SAS is decomposed into some smaller SASs using the binding information; each of the smaller SASs is a schedule for one processor in the platform. Consider an example SDFG with 5 actors denoted by $a_{0}-a_{4}$. Assume $a_{0}, a_{1}$ and $a_{3}$ are bound to processor $P_{1}$ and $a_{2}$ and $a_{4}$ are bound to processor $P_{2}$. Applying the technique of [30] to this imaginary SDFG gives the SAS $s_{0}=\left\langle\left(a_{0}\left(a_{1}{ }^{2} a_{2} a_{3}{ }^{4}\right)^{3}\right)^{2} a_{4}{ }^{5}\right\rangle^{*}$ for the whole SDFG. This SAS can be decomposed using the binding information to form a SAS for each of the processor in the platform. Only considering actors bound to $P_{1}$ in $s_{0}$ results in $s_{01}=\left\langle a_{0}\left(a_{1}^{2} a_{3}^{4}\right)^{3}\right\rangle^{*}$ which is a SAS for the actors bound to $P_{1}$. Similarly, a SAS $s_{02}=\left\langle a_{2}{ }^{6} a_{4}{ }^{5}\right\rangle^{*}$ can be extracted from $s_{0}$ to order actors bound to $P_{2}$. This way we utilize the technique of [30] for multi-processor platforms. The optimality of the generated schedules from the performance or buffer sizing perspective is debatable. However, we use this adapted SAS technique merely to provide some near-optimal inputs to evaluate our SASM schedule encoding technique versus the existing technique. Our techniques do not affect the quality of the scheduling result itself.

\section{B. Comparison on graph sizes}

Table I contains the size of the schedule-extended graphs using the HSDFG-based and DSM techniques to model Lfp and Lrp schedules for a single core platform (see the first two rows of each application line). Using schedules generated by Lfp, the number of decision states is less than when Lrp is used, except in the channel equalizer and MP3 playback applications. By using Lfp scheduling, actors closer to inputs have higher priority compared to actors closer to outputs. This leads to consecutive execution of an actor followed by consecutive execution of another actor with lower priority and so on. Thanks to our optimization in DSM, considering only one decision state before a context switch will be sufficient (e.g., decision state $\omega_{9}$ in Figure 7) and the number of decision states can be reduced significantly. Usually SDFG actors closer to outputs are dependent on actors closer to inputs; this dependency can prevent an actor from being executed consecutively in a graph scheduled by Lrp. As a result, the number of context switches in a graph scheduled by Lrp will typically be larger compared to Lfp. Hence, the effectiveness of the decision state optimization in DSM decreases and extra elements are required to model the schedules in the graph. The exceptions in the channel equalizer and MP3 playback are due to the existence of a cycle in the SDFG; the cycle can increase the number of context switches in the schedule and as a result, Lfp could result in the same or a higher number of decision states in DSM compared to Lrp. However, DSM always outperforms the HSDFG-based technique regardless of the input schedule in our experiments. The number of actors (channels) using DSM is 66\% (71\%) lower compared to the HSDFG-based technique on average and 22\% (17\%) lower in the worst-case observed in our experiments. The average case refers to the mean value of the obtained results and the worstcase reports the smallest graph size reduction (i.e., reduction in numbers of actors and channels compared to the HSDFGbased technique). 
TABLE I

SIZE OF THE SCHEDULE-EXTENDED SDFGS

\begin{tabular}{|c|c|c|c|c|c|c|c|}
\hline \multirow[b]{2}{*}{ Benchmark } & \multicolumn{2}{|l|}{ Orig. size } & \multicolumn{3}{|c|}{ \# actors (\# channels) } & \multicolumn{2}{|c|}{ Reduction compared to the HSDFG-based technique } \\
\hline & \# actors (\# channels) & Schedule type & HSDFG-based & DSM & SASM & DSM & SASM \\
\hline \multirow{3}{*}{ H.263 dec. $[10]$} & & Lfp & $1190(2973)$ & $6(14)$ & NA & $99 \%(99 \%)$ & NA \\
\hline & $4(6)$ & Lrp & $1190(2972)$ & $598(1198)$ & NA & $49 \%(59 \%)$ & NA \\
\hline & & SAS & $1190(2972)$ & $598(1198)$ & $4(12)$ & $49 \%(59 \%)$ & $99 \%(99 \%)$ \\
\hline \multirow{3}{*}{ H.263 enc. [24] } & & Lfp & $201(596)$ & $7(16)$ & NA & $96 \%(97 \%)$ & NA \\
\hline & $5(7)$ & Lrp & 201 (499) & $105(212)$ & NA & $47 \%(57 \%)$ & NA \\
\hline & & SAS & $201(499)$ & $106(214)$ & $5(15)$ & $47 \%(57 \%)$ & $97 \%(97 \%)$ \\
\hline \multirow{3}{*}{ MP3 dec. [10] } & & Lfp & $911(2849)$ & $27(61)$ & NA & $97 \%(97 \%)$ & NA \\
\hline & $14(21)$ & Lrp & 911 (2327) & $400(807)$ & NA & $56 \%(65 \%)$ & NA \\
\hline & & SAS & 911 (2327) & $400(807)$ & $14(44)$ & $56 \%(65 \%)$ & $98 \%(98 \%)$ \\
\hline \multirow{3}{*}{ modem [1] } & & Lfp & $48(115)$ & $22(63)$ & NA & $54 \%(45 \%)$ & NA \\
\hline & $16(35)$ & Lrp & 48 (128) & $35(89)$ & NA & $27 \%(30 \%)$ & NA \\
\hline & & SAS & $48(128)$ & $35(89)$ & $16(61)$ & $27 \%(30 \%)$ & $66 \%(52 \%)$ \\
\hline \multirow{3}{*}{ samplerate conv. [1] } & & Lfp & $612(1639)$ & $12(29)$ & NA & $98 \%(98 \%)$ & NA \\
\hline & $6(11)$ & Lrp & $612(1784)$ & $157(319)$ & NA & $74 \%(82 \%)$ & NA \\
\hline & & SAS & $612(1865)$ & $238(481)$ & $12(31)$ & $61 \%(74 \%)$ & $98 \%(98 \%)$ \\
\hline \multirow{3}{*}{ satellite rec. [25] } & & Lfp & $4515(11638)$ & $41(108)$ & NA & $99 \%(99 \%)$ & NA \\
\hline & $22(48)$ & Lrp & $4515(12820)$ & $1223(2472)$ & NA & $72 \%(80 \%)$ & NA \\
\hline & & SAS & $4515(15270)$ & $3673(7372)$ & $24(91)$ & $18 \%(51 \%)$ & $99 \%(99 \%)$ \\
\hline \multirow{3}{*}{ MP3 playback [26] } & & Lfp & $10601(37531)$ & $5298(10600)$ & NA & $50 \%(71 \%)$ & NA \\
\hline & $4(8)$ & Lrp & 10601 (37529) & $5296(10596)$ & NA & $50 \%(71 \%)$ & NA \\
\hline & & SAS & 10601 (37530) & 5297 (10598) & $6(16)$ & $50 \%(71 \%)$ & $99 \%(99 \%)$ \\
\hline \multirow{3}{*}{ bipartite [25] } & & Lfp & $73(341)$ & $8(20)$ & NA & $89 \%(94 \%)$ & NA \\
\hline & $4(8)$ & Lrp & $73(359)$ & $26(56)$ & NA & $64 \%(84 \%)$ & NA \\
\hline & & SAS & $73(350)$ & $17(38)$ & $9(22)$ & $76 \%(89 \%)$ & $87 \%(93 \%)$ \\
\hline \multirow{3}{*}{ channel eq. [27] } & & Lfp & $41(100)$ & $32(83)$ & NA & $22 \%(17 \%)$ & NA \\
\hline & $21(40)$ & Lrp & $41(95)$ & $27(73)$ & NA & $34 \%(23 \%)$ & NA \\
\hline & & SAS & $41(93)$ & $30(79)$ & $21(65)$ & $27 \%(15 \%)$ & $48 \%(30 \%)$ \\
\hline
\end{tabular}

TABLE II

THE THROUGHPUT ANALYSIS TIME (IN MILLISECONDS)

\begin{tabular}{|c|c|c|c|c|c|c|c|c|}
\hline \multirow[b]{3}{*}{ Benchmark } & \multirow[b]{3}{*}{ \# of processors } & \multicolumn{7}{|c|}{ Type of the schedules / The used schedule modeling technique } \\
\hline & & \multicolumn{3}{|c|}{ SAS } & \multicolumn{2}{|l|}{ Lfp } & \multicolumn{2}{|l|}{ Lrp } \\
\hline & & HSDFG-based & DSM & SASM & HSDFG-based & DSM & HSDFG-based & DSM \\
\hline H.263 dec. & 2 & 36540 & $<1$ & $<1$ & 118720 & $<1$ & 120710 & $<1$ \\
\hline H.263 enc. & 2 & 380 & $<1$ & $<1$ & 690 & $<1$ & 400 & $<1$ \\
\hline MP3 dec. & 3 & 13900 & 320 & 10 & 17660 & $<1$ & 13980 & 1380 \\
\hline modem & 3 & 10 & $<1$ & $<1$ & $<1$ & $<1$ & $<1$ & $<1$ \\
\hline samplerate conv. & 3 & 3970 & 110 & $<1$ & 3140 & $<1$ & 3880 & $<1$ \\
\hline satellite rec. & 2 & not finished in 3 days & 12414400 & 130 & not finished in 3 days & 280 & not finished in 3 days & 675700 \\
\hline MP3 playback & 2 & not finished in 3 days & $<1$ & $<1$ & not finished in 3 days & 10780 & not finished in 3 days & $<1$ \\
\hline bipartite & 2 & 30 & $<1$ & $<1$ & 40 & $<1$ & 50 & $<1$ \\
\hline channel eq. & 3 & $<1$ & $<1$ & $<1$ & $<1$ & $<1$ & $<1$ & $<1$ \\
\hline
\end{tabular}

SASs are a suitable class of schedules that minimize code memory size. DSM is able to model any arbitrary schedule in an SDFG. SAS can be modeled using DSM; however, it is possible to consider the intrinsic property of SASs when modeling a SAS in an SDFG. Our second technique, SASM, uses the fact that each actor appears only once in the looped schedule form. SASM models the counter concept in the graph in order to force actors to be executed a specific number of times. The third row of each application line in Table I contains the size of the schedule-extended graphs using the HSDFGbased, DSM and SASM techniques to model SASs, generated by the technique developed in [30].

SASM results in a schedule-extended SDFG with a limited number of extra actors and channels. For example, SASM only adds 2 (8) extra actors (channels) to the original graph of the MP3 playback application in order to model a SAS, while the HSDFG-based technique adds 1057 (37522) extra actors (channels) to model the same schedule. The graphs obtained by SASM have $88 \%$ (85\%) and $48 \%$ (30\%) less actors (channels) compared to the HSDFG-based technique on average and in the worst-case among the benchmark applications. Using the DSM technique to model the same SASs results in $46 \%$ $(57 \%)$ and $27 \%(15 \%)$ less actors (channels) compared to the
HSDFG-based technique on average and in the worst-case. Our results confirm that the techniques proposed in this paper achieve a more compact schedule-extended graph compared to the available technique.

\section{Comparison on analysis times}

The time required to perform an analysis on an SDFG depends on the size of the graph and the number of cycles in the graph. As an example, the throughput analysis of [9] is performed on the schedule-extended graphs using our techniques and the HSDFG-based technique. Our experiments are performed to evaluate the impact of the graph size on the analysis time of a common analysis technique. Note that other techniques (e.g., YTO [31]) can be employed to calculate throughput of HSDFGs, but the size of the schedule-extended HSDFGs is such that our conclusions remain the same. The benchmark graphs are mapped onto multi-processor platforms with two or three processors. Table II contains the throughput analysis times when SASs, list forward priority (Lfp) schedules and list reverse priority (Lrp) schedules are used as input schedules. The results show the superiority of SASM over DSM and the HSDFG-based technique. Note that the SDFG 


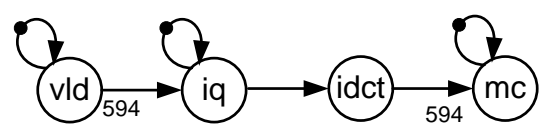

Fig. 13. SDFG of H.263 decoder application.

to HSDFG conversion is fast; the numbers reported for the HSDFG-based technique in Table II are related to the runtime of the throughput analysis from [9]. In our experiment, the run-time of a throughput calculation for HSDFGs is long independent of the analysis technique used (i.e., state-space [9], YTO [31], etc.).

\section{Comparison on buffer sizes}

To further analyze the effectiveness of our techniques, the buffer sizing algorithm from [10] is applied to the scheduleextended SDFGs of the H.263 decoder and MP3 decoder. Figure 13 depicts the SDFG of the H.263 decoder. Besides the compactness of the schedule-extended graph, our techniques preserve the original structure of an SDFG (when ignoring the added actors and channels), allowing accurate buffer sizing, which is not guaranteed for the state of the art technique. The H.263 decoder is mapped to a platform with two processors. The actors $v l d$ and $i q$ are mapped to the first processor with a PSOS $\left\langle v l d(i q)^{594}\right\rangle^{*}$ and the actor $i d c t$ and $m c$ are mapped to the second processor with a PSOS $\left\langle(i d c t)^{594} m c\right\rangle^{*}$. The analysis time for buffer sizing on the schedule-extended H.263 decoder is less than $1 \mathrm{~ms}$ when using DSM (or SASM) to model the schedules. The same analysis takes $1330 \mathrm{~ms}$ when using the technique from [12] to model the same schedules in the same graph. Figure 14(a) shows the Pareto space of throughput and buffer size when modeling the schedules with DSM (or SASM) and the HSDFG-based technique [12]. In this experiment, the schedules are first modeled in the graph; then, the buffer sizing technique of [10] is applied. A single channel in an SDFG corresponds to a set of channels in the equivalent HSDFG. As a result, the buffer sizing technique cannot find the minimal buffer size when applying it on the equivalent HSDFG. Our experiments show these inaccuracies. Applying buffer sizing on the graph which models the schedules using the technique from [12] results in $49 \%$ overestimation in required buffers compared to applying the same buffer sizing technique on the graph which models the schedules with one of our techniques. Note that the maximal achievable throughput is independent of the way schedules are encoded. The analysis results confirm this. Only the computed buffer sizes differ. For instance in both cases of Figure 14, the maximal throughput for the given schedules is always achievable, also by using the HSDFG-based schedule modeling technique; the latter suggests the need for larger buffers though. Figure 14(b) shows results for the MP3 decoder. We use the mapping and scheduling from [32] for a platform with 3 processors. The analysis time on the graph which models the schedule using one of our techniques is $594 \mathrm{~ms}$ while $141610 \mathrm{~ms}$ is required to perform the same analysis on the graph using the technique from [12]. Using the technique from [12] results

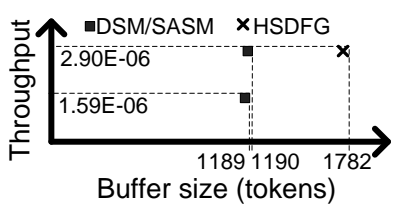

(a) H.263 decoder

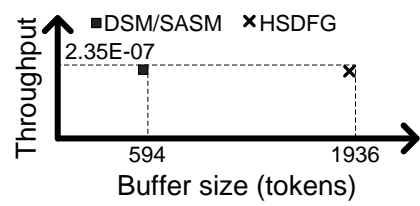

(b) MP3 decoder
Fig. 14. Pareto space of schedule-extended graphs modeled by DSM and HSDFG-based techniques (the scales of the two graphs are different).

in $226 \%$ overestimation in buffer size compared to using our techniques.

Modeling a PSOS in an SDFG using DSM requires execution of one complete SDFG iteration. The number of states in one iteration could be exponential in the number of actors in the graph. However, for all real-world SDFGs used in our experiments, the execution time of DSM is below 1 ms. SASM also models SASs based on the structure of the schedules in their looped form; as each actor appears once in a SAS, the complexity of SASM depends on the number of actors in the graph. Similar to DSM, the execution time of SASM is always below $1 \mathrm{~ms}$ in our experiments. The complexity of our techniques relates to the length of the SDFG iteration and the number of processors in the platform (i.e., $|P|)$. Hence, the complexity of our techniques is bounded to $O\left(|P| \cdot \sum_{a \in A} \gamma(a)\right)$.

\section{CONCLUSION}

We present two techniques, DSM and SASM, to model PSOSs and SASs directly in an SDFG. The resulting graphs are much smaller (often much less than half the size) than graphs resulting from the state of the art technique that first converts an SDFG to an HSDFG. This results in a speedup of analysis techniques. Computing the trade-off between buffering and throughput for multi-processor platforms, for example, becomes several orders of magnitude faster. Moreover, properties like buffer sizes can be analyzed more accurately. The techniques have been integrated in the $\mathrm{SDF}^{3}$ tool set available at http://www.es.ele.tue.nl/sdf3. This allows easy integration of the techniques in multi-processor design flows.

\section{ACKNOWLEDGMENT}

We thank the reviewers for their constructive comments, which helped improving the presentation of the paper. This work was supported in part by the Dutch Technology Foundation STW, project NEST 10346.

\section{REFERENCES}

[1] S. S. Bhattacharyya et al., "Synthesis of embedded software from synchronous dataflow specifications," Journal of VLSI Signal Processing, vol. 21, pp. 151-166, 1999.

[2] S. Sriram et al., Embedded Multiprocessors: Scheduling and Synchronization, 2nd ed. CRC Press, 2009.

[3] P. Poplavko et al., "Task-level timing models for guaranteed performance in multiprocessor networks-on-chip," CASES. ACM, 2003, pp. 63-72.

[4] M.-Y. Ko et al., "Compact procedural implementation in DSP software synthesis through recursive graph decomposition," SCOPES. ACM, 2004, pp. 47-61.

[5] A. Bonfietti et al., "Throughput constraint for synchronous data flow graphs," CPAIOR. Springer-Verlag, 2009, pp. 26-40. 
[6] S. Stuijk et al., "Multiprocessor resource allocation for throughputconstrained synchronous dataflow graphs," in DAC, 2007, pp. 777-782.

[7] W. Liu et al., "Efficient SAT-based mapping and scheduling of homogeneous synchronous dataflow graphs for throughput optimization," RTSS. IEEE, 2008, pp. 492-504.

[8] Y. Yang et al., "Automated bottleneck-driven design-space exploration of media processing systems," DATE. ACM, 2010, pp. 1041-1046.

[9] A. Ghamarian et al., "Throughput analysis of synchronous data flow graphs," ACSD. IEEE, 2006, pp. 25-36.

[10] S. Stuijk et al., "Throughput-buffering trade-off exploration for cyclostatic and synchronous dataflow graphs," IEEE Transaction on Computers, vol. 57, no. 10, pp. 1331-1345, 2008.

[11] M. Benazouz et al., "A new method for minimizing buffer sizes for cyclo-static dataflow graphs," in ESTIMedia'10. IEEE, pp. $11-20$.

[12] N. Bambha et al., "Intermediate representations for design automation of multiprocessor DSP systems," Design Automation for Embedded Systems, vol. 7, no. 4, pp. 307-323, 2002.

[13] M. Damavandpeyma et al., "Modeling static-order schedules in synchronous dataflow graphs," in DATE'12. ACM, pp. 775-780.

[14] A.-P. Wang et al., "Buffer optimization and dispatching scheme for embedded systems with behavioral transparency," ACM Transactions on Design Automation of Electronic Systems, vol. 17, no. 4, pp. 41:1-41:26, Oct. 2012.

[15] M. Damavandpeyma et al., "Throughput-constrained DVFS for scenarioaware dataflow graphs," in RTAS'13. IEEE.

[16] M. H. Wiggers et al., "Monotonicity and run-time scheduling," EMSOFT. ACM, 2009, pp. 177-186.

[17] D. Thiele et al., "Optimizing performance analysis for synchronous dataflow graphs with shared resources," in DATE'12. ACM, pp. 635640.

[18] R. Henia et al., "System level performance analysis - the SymTA/S approach," IEE Proccedings Computers and Digital Techniques, vol. 152 , no. 2 , pp. 148 - 166, mar 2005

[19] L. Thiele et al., "Real-time calculus for scheduling hard real-time systems," in ISCAS'00, vol. 4. IEEE, 2000, pp. $101-104$.

[20] H. H. Wu et al., "A model-based schedule representation for heterogeneous mapping of dataflow graphs," HCW. IEEE, 2011, pp. 66-77.

[21] S. Bhattacharyya et al., Software Synthesis from Dataflow Graphs. Kluwer Academic Publishers, 1996.

[22] E. Lee et al., "Synchronous data flow," Proceeding of the IEEE, vol. 75, no. 9, pp. 1235-1245, 1987.

[23] M. Geilen et al., "Minimising buffer requirements of synchronous dataflow graphs with model checking," DAC '05. ACM, 2005, pp. 819-824.

[24] H. Oh et al., "Fractional rate dataflow model for efficient code synthesis," Journal of VLSI Signal Processing, vol. 37, pp. 41-51, 2004.

[25] S. Ritz et al., "Scheduling for optimum data memory compaction in block diagram oriented software synthesis," ICASSP. IEEE, 1995

[26] M. H. Wiggers et al., "Efficient computation of buffer capacities for cyclo-static dataflow graphs," DAC. ACM, 2007, pp. 658-663.

[27] A. Moonen et al., "Practical and accurate throughput analysis with the cyclo static dataflow model," MASCOTS. IEEE, 2007, pp. 238-245.

[28] S. Stuijk et al., "SDF': SDF for free," ACSD. IEEE, 2006, pp. 276278.

[29] G. De Micheli, Synthesis and Optimization of Digital Circuits, 1st ed. McGraw-Hill, 1994.

[30] P. K. Murthy et al., "Joint minimization of code and data for synchronous dataflow programs," Formal Methods in System Design, vol. 11, no. 1, pp. 41-70, 1997.

[31] N. E. Young et al., "Faster parametric shortest path and minimum balance algorithms," CoRR, vol. cs.DS/0205041, 2002.

[32] M. Geilen et al., "Worst-case performance analysis of synchronous dataflow scenarios," CODES+ISSS. ACM, 2010, pp. 125-134.

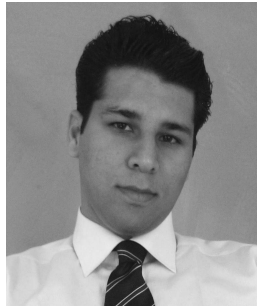

Morteza Damavandpeyma received his B.Sc. and M.Sc. degrees in computer engineering from the University of Tehran, Iran in 2006 and 2008, respectively. $\mathrm{He}$ is currently a Ph.D. candidate in the Department of Electrical Engineering at the Eindhoven University of Technology (TU/e). His research interests include modeling, analysis and synthesis of embedded systems and multiprocessor System-on-Chips.

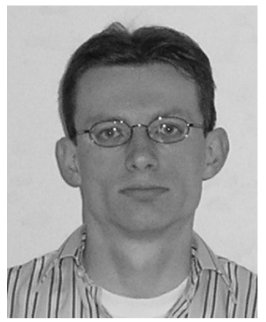

Sander Stuijk received his M.Sc. degree (with honors) in Electrical Engineering in 2002 and his $\mathrm{Ph}$.D. degree in 2007 from the Eindhoven University of Technology. He is currently an assistant professor in the Department of Electrical Engineering at the Eindhoven University of Technology. His research interests include modeling methods and mapping techniques for the design, specification, analysis and synthesis of predictable hardware/software systems.

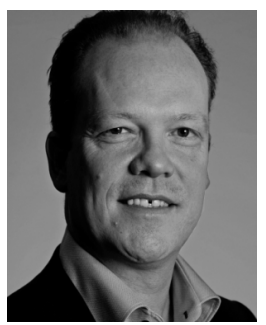

Twan Basten is a professor in the Electrical Engineering department at Eindhoven University of Technology (TU/e), the Netherlands, where he chairs the Electronic Systems group. He is also a senior research fellow of TNO Embedded Systems Innovation in the Netherlands. He holds an M.Sc. and a Ph.D. in Computing Science from TU/e. His research interests include embedded and cyberphysical systems, dependable computing and computational models.

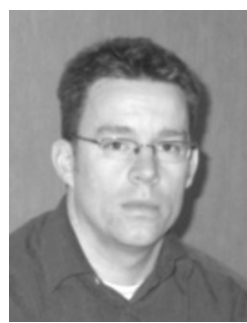

Marc Geilen is an assistant professor in the Department of Electrical Engineering at Eindhoven University of Technology. He holds an M.Sc. in Information Technology and a Ph.D. from the Eindhoven University of Technology. His research interests include modeling, simulation and programming of multimedia systems, multiprocessor systemson-chip, networked embedded systems and cyberphysical systems, and multi-objective optimization and trade-off analysis.

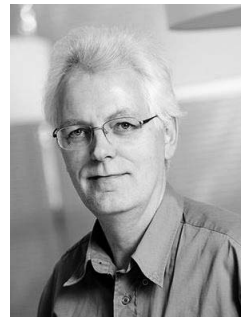

Henk Corporaal has gained an M.Sc. in Theoretical Physics from the University of Groningen, and a Ph.D. in Electrical Engineering, in the area of Computer Architecture, from Delft University of Technology. He is a professor in Embedded System Architectures at the Eindhoven University of Technology (TU/e) in The Netherlands. His current research projects are on low power single and multiprocessor architectures, their programmability, and the predictable design of soft- and hard real-time systems. 


\section{APPENDIX A}

\section{CORRECTNESS OF DSM}

This section discusses the correctness of DSM in modeling a single PSOS for a sub-set of the actors of the SDFG. The extra actors and channels added by our techniques to model a PSOS (e.g., $s_{i}$ ) are only placed between actors of that schedule (i.e., $A_{i}$ ) and this is not imposing any restriction on the other actors of the SDFG (i.e., actors in $A \backslash A_{i}$ ). Hence, if we can model a single PSOS in the SDFG, then we can model multiple PSOSs by applying the algorithm multiple times.

DSM adds some actors, denoted by $A_{s_{i}}$, and channels, denoted by $C_{s_{i}}$ to model the PSOS $s_{i}$ in SDFG $G(A, C)$. $G^{\prime}\left(A^{\prime}, C^{\prime}\right)$ is the SDFG that models $s_{i}$ in $G$ using DSM where $A^{\prime}=A \cup A_{s_{i}}$ and $C^{\prime}=C \cup C_{s_{i}}$.

The existence of a repetition vector for an SDFG $G(A, C)$ ensures a balance between production and consumption rates. Hence, a balance equation can be defined as follow for each channel $c=(p, q) \in C$ where $p$ and $q$ are ports of actors $a_{p}$ and $a_{q}$ respectively: Rate $(p) \cdot \gamma\left(a_{p}\right)=\operatorname{Rate}(q) \cdot \gamma\left(a_{q}\right)$. The existence of a repetition vector $\gamma$ for an SDFG ensures that each balance equation related to a channel in the SDFG holds; under this situation the SDFG is consistent. The following proposition shows the consistency of the schedule-extended SDFG $G^{\prime}$.

Proposition 1. The SDFG $G^{\prime}\left(A^{\prime}, C^{\prime}\right)$ which models PSOS $s_{i}$ in the consistent $\operatorname{SDFG} G(A, C)$ is consistent.

Proof. The SDFG $G$ is consistent. Hence, a non-trivial repetition vector $\gamma$ exists for $G$. We need to show that a non-trivial repetition vector $\gamma^{\prime}$ exists for $G^{\prime}$. The balance equation related to each self-loop added by DSM (in line 1 of Algorithm 1) to remove auto-concurrency is always valid because the source and destination actor of the self-loop channel are identical with production and consumption rate equal to 1 . The rates of the other channels added by DSM (for decision states or inter-iteration execution) share the following properties: (1) the newly added channel $c \in C_{s_{i}}$, which is added by DSM (in all lines 7, 8, 16 or 18 of Algorithm 1), is between an actor $a_{p} \in A\left(=A^{\prime} \backslash A_{s_{i}}\right)$ and an actor $a_{q} \in A_{s_{i}}$; (2) the rate of the new channel on the side of the actor $a_{q}$ is equal to the count of the actor $a_{p}$ in one iteration of $s_{i}$ (i.e., $\left.C N T\left(a_{p}, s_{i}\right)\right)$; (3) the rate of the new channel on the side of the actor $a_{p}$ is equal to 1 . From these properties we conclude that $a_{q} \in A_{s_{i}}$, which is added by DSM (in both line 6 and 13 of Algorithm 1), fires only once in each iteration of $s_{i}$. Consider $a_{q}$ as the producer (see Figure 15(a)) of the newly added channel (i.e., $\left.\left(a_{q}, a_{p}\right) \in C_{s_{i}}\right)$; then, the only firing of $a_{q}$ in one iteration of $s_{i}$ provides $C N T\left(a_{p}, s_{i}\right)$ tokens for all firings of $a_{p}$ in one iteration of $s_{i}$. Now reconsider actor $a_{q}$ as the consumer (see Figure 15(b)) of the newly added channel (i.e., $\left(a_{p}, a_{q}\right) \in C_{s_{i}}$ ); as a result, all firings of actor $a_{p}$ in one iteration of $s_{i}$ provide $C N T\left(a_{p}, s_{i}\right)$ tokens for only one firing of $a_{q}$.

Let set $A_{i}$ be the set of the actors in the PSOS $s_{i}$. Consider that each actor $a_{p} \in A_{i}$ appears $r \cdot \gamma\left(a_{p}\right)$ times in the PSOS $s_{i}\left(r=\frac{u}{v}\right.$ where $\left.u, v \in \mathbb{N}\right)$ where the value $r$ is identical for all actors in $s_{i}$. The appearance count of the actor $a_{p} \in$
$A_{i}$ in $s_{i}$ is represented by $C N T\left(a_{p}, s_{i}\right)$ and it is assumed to be equal to $\frac{u}{v} \cdot \gamma\left(a_{p}\right)$. We can write the above statement as follow: $C N T\left(a_{p}, s_{i}\right) \cdot v=\gamma\left(a_{p}\right) \cdot u$. From this equation we can conclude that $u$ iterations of the SDFG $G$ cause $v$ iterations of the PSOS $s_{i}$, leading to $v$ firings of each of the actors added by DSM (i.e., actors from the set $A_{s_{i}}$ ). So, in $u$ iterations of the SDFG $G$ (or $v$ iterations of the PSOS $s_{i}$ ) the following equation holds for each channel $\left(a_{p}, a_{q}\right) \in C_{s_{i}}$ or $\left(a_{q}, a_{p}\right) \in C_{s_{i}}$ (where $a_{p} \in A_{i}$ and $\left.a_{q} \in A_{s_{i}}\right)$ :

$$
\underbrace{C N T\left(a_{p}, s_{i}\right)}_{\text {Rate }\left(a_{q}\right)} \cdot \underbrace{v}_{\gamma^{\prime}\left(a_{q}\right)}=\underbrace{1}_{\text {Rate }\left(a_{p}\right)} \cdot \underbrace{\gamma\left(a_{p}\right) \cdot u}_{\gamma^{\prime}\left(a_{p}\right)}
$$

The entries of the repetition vector of the schedule-extended graph can be obtained using Equation 1. So a non-trivial repetition vector $\gamma^{\prime}$ can be found for the schedule-extended SDFG $G^{\prime}$. The relation between the repetition vector of the schedule-extended SDFG and the repetition vector of the original graph is as follows: $\gamma^{\prime}\left(a_{q}\right)$ (where $\left.a_{q} \in A_{s_{i}}\right)$ is equal to $v$ and $\gamma^{\prime}\left(a_{p}\right)$ (where $a_{p} \in A$ ) is equal to $\gamma\left(a_{p}\right) \cdot u$.

The following proposition states that inter-iteration execution for actors of a PSOS $s_{i}$ is impossible in the SDFG $G^{\prime}$ which extends the original SDFG $G$ with PSOS $s_{i}$ using DSM.

Proposition 2. PSOS inter-iteration execution is impossible for any actor appearing in PSOS $s_{i}=\left\langle\alpha_{1} \alpha_{2} \ldots \alpha_{n}\right\rangle^{*}$ in the SDFG $G^{\prime}\left(A^{\prime}, C^{\prime}\right)$, which models PSOS $s_{i}$ in the SDFG $G(A, C)$ using DSM.

Proof. Let set $A_{i}$ be the set of the actors in the PSOS $s_{i}$. In one iteration of a PSOS $s_{i}$, an actor $a_{p} \in A_{i}$ could be enabled more often than its designated number (i.e., $C N T\left(a_{p}, s_{i}\right)$ times). DSM prevents this by creating a dependency from the last actor appearing in $s_{i}$ (i.e., actor $a_{L}=\alpha_{n}$ ) to the first actor appearing in $s_{i}$ (i.e., actor $a_{F}=\alpha_{1}$ ) (see lines 4-8 in DSM). This dependency is created by inserting a new actor $a_{i . e n d}$ and two channels $c_{i \text {.pre }}$ and $c_{i . p r o}$. The source (destination) actor of the channel $c_{i . p r e}$ is $a_{L}\left(a_{i . e n d}\right)$ with rate $1\left(C N T\left(a_{L}, s_{i}\right)\right)$. So, one firing of $a_{i . e n d}$ needs $C N T\left(a_{L}, s_{i}\right)$ tokens available in $c_{i \text {.pre }}$; for this purpose actor $a_{L}$ should fire $C N T\left(a_{L}, s_{i}\right)$ times. The destination actor of the channel $c_{i . p r o}$ is $a_{F}$; the consumption rate of $a_{F}$ from $c_{i \text {.pro }}$ is 1 . The source actor of the channel $c_{i . p r o}$ is $a_{i . e n d}$; the production rate of $a_{i . \text { end }}$ to $c_{i . p r o}$ is $C N T\left(a_{F}, s_{i}\right)$. So the firing of $a_{F}$ related to one iteration of PSOS $s_{i}$ needs $C N T\left(a_{F}, s_{i}\right)$ tokens available in $c_{i . p r o}$; for this purpose actor $a_{i . e n d}$ should fire once. The channel $c_{i . p r o}$ is initialized with $C N T\left(a_{F}, s_{i}\right)$ number of tokens; this number of tokens provides enough tokens for actor $a_{F}$ to fire $C N T\left(a_{F}, s_{i}\right)$ times in one iteration of PSOS $s_{i}$. The subsequent firing of actor $a_{F}$ depends on the firing of the actor $a_{i . e n d}$ and the firing of the actor $a_{i . e n d}$ demands $C N T\left(a_{L}, s_{i}\right)$ times a firing of actor $a_{L}$. Hence, the firing of $a_{F}$ belonging

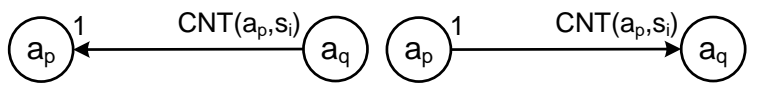
(a) $a_{q}$ is producer
(b) $a_{q}$ is consumer

Fig. 15. Extra actor $a_{q}$ added by DSM in different situations. 
to the subsequent iteration of $s_{i}$ can be performed only after $a_{L}$ finishes all of its firings belonging to the current iteration of $s_{i}$ (i.e., after completion of the current iteration of $s_{i}$ ). This holds because actor $a_{F}$ is the first actor which should be fired in an iteration of $s_{i}$ and other actors in $s_{i}$ cannot get enabled before the first firing of $a_{F}$. This second fact is guaranteed by adding decision state constructs (i.e., lines 12-18 in DSM) for any possible decision state and Proposition 6 below.

Even after eliminating inter-iteration execution from the SDFG, multiple actors from a schedule may be enabled in an SDFG iteration. We call such a state a decision state. The following proposition shows that analyzing only one SDFG iteration is enough to identify all possible decision states.

Proposition 3. Executing an $\operatorname{SDFG} G(A, C)$ for one iteration is enough to find all decision states within a PSOS $s_{i}$.

Proof. Let the set $A_{i}$ be the set of the actors in $s_{i}$ and $A_{o}=A \backslash A_{i}$ the remaining actors in $A$. Consider interprocessor channels, which are defined as channel originating from the actors in $A_{o}$ to the actors in $A_{i}$ denoted by $C_{i p c}=\left\{\left(a_{p}, a_{q}\right) \in C \mid a_{p} \in A_{o} \wedge a_{q} \in A_{i}\right\}$. The execution of an actor $a_{p} \in A_{o}$ where $\left(a_{p}, a_{q}\right) \in C_{i p c}$ up-to its entry in the repetition vector of the SDFG produces $\gamma\left(a_{p}\right) \cdot \operatorname{Rate}\left(a_{p}\right)$ tokens in the corresponding channel $\left(a_{p}, a_{q}\right) \in C_{i p c}$. Actor $a_{q} \in A_{i}$ consumes those produced tokens within one iteration of the normalized PSOS $s_{i}$ (because $\gamma\left(a_{p}\right) \cdot \operatorname{Rate}\left(a_{p}\right)=$ $\gamma\left(a_{q}\right)$. Rate $\left.\left(a_{q}\right)\right)$. Hence, actors in $A_{i}$ can receive the required tokens from all inter-processor channels $C_{i p c}$ for one iteration of the normalized PSOS $s_{i}$. This means that all possible decision states related to PSOS $s_{i}$ are detectable.

Actors in $A_{o}$ could possibly fire more than the number mentioned above (i.e., corresponding value in vector $\gamma$ ) if the channel dependencies in the SDFG allow additional firings of these actors. This could cause more than enough tokens (for one iteration of the normalized PSOS $s_{i}$ ) in channels $C_{i p c}$. This could enable an actor in $A_{i}$ more than its designated number in one iteration of the normalized PSOS $s_{i}$. To avoid this undesirable actor enabling, some constructs are added to the graph to prevent inter-iteration execution (see Proposition 2). As a result, extra tokens produced by further firing of the actors $A_{o}$ cannot enable any actor in $A_{i}$ more than its designated value in one iteration of the normalized PSOS $s_{i}$. So, executing actors in the SDFG up-to their repetition vector entry (i.e., one SDFG iteration) is enough to find all decision states within $s_{i}$.

The identified decision states may be redundant. Proposition 4 discuses the proposed decision state reduction in the DSM.

Proposition 4. Let $\sigma$ be an execution of an $\operatorname{SDFG}(A, C)$ and a PSOS $s_{i}$ which schedules actors $A_{i} \subseteq A$. In the execution $\sigma$, consider y consecutive decision states $\omega_{x+1}, \omega_{x+2}, \cdots, \omega_{x+y}$. Assume that $a_{o} \in A_{i}$ is an opponent actor in each of these decision states but not the actor of choice in any of them. It is sufficient to only consider the last decision state $\omega_{x+y}$ to postpone the firing of the opponent actor $a_{o}$ in those consecutive decision states to the state $\omega_{x+y+1} \in \sigma$.
Proof. The purpose of the components added by DSM (i.e., lines 13-18 in Algorithm 1) in a decision state $\omega_{j} \in \Omega$ is to prevent any opponent actor $a_{o}$ which is not the actor of choice in decision state $\omega_{j}$ from getting enabled in that state and as such to postpone that firing to the state $\omega_{j+1}$. It is assumed that the opponent actor $a_{o}$ is enabled in the consecutive decision states $\omega_{x+1}, \omega_{x+2}, \cdots, \omega_{x+y}$. Suppose that the opponent actor $a_{o}$ was fired $e$ times before the first decision state (i.e., $\omega_{x+1}$ ) where $0 \leq e<\gamma\left(a_{o}\right)$. The components added by DSM in the last decision state $\omega_{x+y}$ prevent the opponent actor $a_{o}$ from getting enabled for the $(e+1)^{t h}$ time in decision state $\omega_{x+y}$. As a result, the opponent actor $a_{o}$ can also not be enabled in states $\omega_{x+1}, \omega_{x+2}, \cdots, \omega_{x+y-1}$ after adding DSM components for decision state $\omega_{x+y}$. So, the components added by DSM in the last decision state of consecutive decision states are enough to prevent the firing of an opponent actor which is not the actor of choice in those consecutive decision states.

Decision state folding overlaps the consecutive repetitions of the designated PSOS in an SDFG iteration to reduce the number of decision states. The following proposition states that decision state folding does not dismiss any decision state.

Proposition 5. Consider PSOS $s_{i}=\left\langle\alpha_{1} \alpha_{2} \ldots \alpha_{n}\right\rangle^{*}$ for the sub-set of actors $A_{i}$ from $\operatorname{SDFG}(A, C)$; assume $s_{i}$ is repeated $\mu_{i}$ times to form the corresponding normalized PSOS $\left(s_{i}^{\prime}=\right.$ $\left.\left\langle\left(s_{i}\right)^{\mu_{i}}\right\rangle^{*}\right)$ to identify decision states related to PSOS $s_{i}$. After decision state folding all decision states are preserved.

Proof. Normalization can be done by repeating $s_{i} \mu_{i}$ times $\left(\mu_{i}\right.$ is the normalization factor of $\left.s_{i}\right)$. Decision state identification is applied to the normalized PSOS $s_{i}^{\prime}=$ $\left\langle\left(s_{i}\right)^{\mu_{i}}\right\rangle^{*}=\langle\underbrace{\alpha_{1} \alpha_{2} \ldots \alpha_{n}}_{1^{\text {st }}} \underbrace{\alpha_{1} \alpha_{2} \ldots \alpha_{n}}_{2^{\text {nd }}} \cdots \underbrace{\alpha_{1} \alpha_{2} \ldots \alpha_{n}}_{\mu_{i}{ }^{t h}}\rangle^{*}$. Decision point constructs are added based on the given PSOS $s_{i}$. Folding groups the identified decision states of the normalized PSOS $s_{i}^{\prime}$ in the following manner: the state related to the firing of an actor $\alpha_{j}$ in $s_{i}$ is considered as decision state if a decision state is found at least in one of the $\mu_{i}$ states that $\alpha_{j}$ fires in the execution related to $s_{i}^{\prime}$.

The corresponding state related to firing of the actor $\alpha_{j}$ in $s_{i}$ which is considered as a decision state imposes a decision state to all $\mu_{i}$ corresponding states of actor $\alpha_{j}$ in $s_{i}^{\prime}$. So, no decision state will be lost after decision state folding and the only effect is introducing unnecessary decision state controlling. We need to show that this extra controlling does not affect the execution of the SDFG according to the schedule. The construct added in a decision state is used to guarantee execution of the actor of choice of that decision state. Even if a state is not a decision state, the added constructs for that unnecessary decision state only ensure that the only enabled actor in that state (i.e., the actor of choice) can be fired. This does not violate the actor firing order according to the PSOS in that state.

DSM adds some components per decision state to enforce the firing of the enabled actor in a decision state which is in line with the given PSOS (i.e., actor of choice). Proposition 6 explains how those components can guarantee the firing of the actor of choice in the decision state. 
Proposition 6. The PSOS $s_{i}$ is a schedule for actors $A_{i} \subseteq A$ from $\operatorname{SDFG}(A, C)$. Let $\omega_{j} \in \Omega$ be a decision state within PSOS $s_{i}$ and $\Delta_{j} \subseteq A_{i}$ the set of the opponent actors in decision state $\omega_{j}$. The actor of choice in decision state $\omega_{j}$ (denoted by actor $a_{c}$ ) is the only actor which can fire in decision state $\omega_{j}$ among all actors in $\Delta_{j}$ after applying DSM. Also, one iteration of the resulting schedule-extended SDFG resets the tokens to their initial positions (i.e., the periodic behavior of the original SDFG is also preserved).

Proof. We need to show that the opponent actors $\Delta_{j} \backslash\left\{a_{c}\right\}$ can not be enabled in the decision state $\omega_{j}$ after applying DSM. Accordingly, the actor of choice $a_{c}$ in the decision state $\omega_{j}$ is the only actor which can fire in $\omega_{j}$ among all actors in $\Delta_{j}$.

In the DSM technique, actor $a_{i . \omega_{j}}$ is added for each decision state $\omega_{j} \in \Omega$ within the PSOS $s_{i}$. An opponent actor $a_{k} \in \Delta_{j} \backslash\left\{a_{c}\right\}$ in the decision state $\omega_{j}$ is dependent on the new actor $a_{i . \omega_{j}}$ because of the added channel $c_{i . a_{k} \omega_{j}}$; this channel is initialized with $\operatorname{BEF}\left(a_{k}, \operatorname{pos}\left[\omega_{j}\right], s_{i}\right)$ tokens. The rate of the channel $c_{i . a_{k} \omega_{j}}$ on the side of $a_{k}\left(a_{i . \omega_{j}}\right)$ is equal to one $\left(C N T\left(a_{k}, s_{i}\right)\right)$. The new actor $a_{i . \omega_{j}}$ is also dependent on the actor of choice $a_{c}$ of the decision state $\omega_{j}$ because of the added channel $c_{i . a_{c} \omega_{j}}$; this channel is initialized with $A F T\left(a_{c}, \operatorname{pos}\left[\omega_{j}\right], s_{i}\right)$ tokens. The rate of the channel $c_{i . a_{c} \omega_{j}}$ on the side of $a_{c}\left(a_{i . \omega_{j}}\right)$ is equal to one $\left(C N T\left(a_{c}, s_{i}\right)\right)$.

An opponent actor $a_{k} \in \Delta_{j} \backslash\left\{a_{c}\right\}$ fires $B E F\left(a_{k}, \operatorname{pos}\left[\omega_{j}\right], s_{i}\right)$ times before decision state $\omega_{j}$ and every time the opponent actor $a_{k}$ consumes one token from $c_{i, a_{k} \omega_{j}}$. So, the $B E F\left(a_{k}, \operatorname{pos}\left[\omega_{j}\right], s_{i}\right)$ firings of $a_{k}$ before $\omega_{j}$ consume all tokens which were available in channel $c_{i . a_{k} \omega_{j}}$. Hence, the opponent actor $a_{k} \in \Delta_{j} \backslash\left\{a_{c}\right\}$ cannot fire in $\omega_{j}$. Firings of the opponent actor $a_{k} \in \Delta_{j} \backslash\left\{a_{c}\right\}$ from decision state $\omega_{j}$ onward will be dependent on the firing of $a_{i . \omega_{j}}$ to provide the required tokens in channel $c_{i . a_{k} \omega_{j}}$. As mentioned before, $a_{i . \omega_{j}}$ depends on the actor of choice $a_{c}$. So, the opponent actor $a_{k} \in \Delta_{j} \backslash\left\{a_{c}\right\}$ cannot fire from decision state $\omega_{j}$ onward, unless the actor of choice $a_{c}$ of the decision state $\omega_{j}$ fires. Hence, the actor of choice $a_{c}$ is the only actor among the other opponent actors in $\omega_{j}$ which can fire. The actor of choice $a_{c}$ is fired $B E F\left(a_{c}, \operatorname{pos}\left[\omega_{j}\right], s_{i}\right)$ times by state $\omega_{j}$ and this results in $B E F\left(a_{c}, \operatorname{pos}\left[\omega_{j}\right], s_{i}\right)$ tokens being produced in channel $c_{i . a_{c} \omega_{j}}$; as channel $c_{i . a_{c} \omega_{j}}$ is initialized with $\operatorname{AFT}\left(a_{c}, \operatorname{pos}\left[\omega_{j}\right], s_{i}\right)$ tokens, the number of tokens in this channel is $\operatorname{BEF}\left(a_{c}, \operatorname{pos}\left[\omega_{j}\right], s_{i}\right)+\operatorname{AFT}\left(a_{c}, \operatorname{pos}\left[\omega_{j}\right], s_{i}\right)=C N T\left(a_{c}, s_{i}\right)$ after firing $a_{c}$ in decision state $\omega_{j}$. So, there will be sufficient tokens (for one firing of $a_{i . \omega_{j}}$ ) on the only channel leading to $a_{i . \omega_{j}}$ after firing of the actor of choice $a_{c}$ in decision state $\omega_{j}$. Then, firing of $a_{i . \omega_{j}}$ consumes all $C N T\left(a_{c}, s_{i}\right)$ tokens that are present in channel $c_{i . a_{c} \omega_{j}}$ and it produces $C N T\left(a_{k}, s_{i}\right)$ tokens in channel $c_{i . a_{k} \omega_{j}}\left(a_{k} \in \Delta_{j} \backslash\left\{a_{c}\right\}\right)$; therefore, the opponent actors $\Delta_{j} \backslash\left\{a_{c}\right\}$ are not any more dependent on $a_{i . \omega_{j}}$ in the remainder of the current iteration of $s_{i}$.

The firings of the opponent actor $a_{k} \in \Delta_{j} \backslash\left\{a_{c}\right\}$ after decision state $\omega_{j}$ consumes $A F T\left(a_{k}, \operatorname{pos}\left[\omega_{j}\right], s_{i}\right)$ tokens from channel $c_{i . a_{k} \omega_{j}}$; as a result, at the end of the PSOS iteration, the number of tokens on this channel returns to its initial value which is
$B E F\left(a_{k}, \operatorname{pos}\left[\omega_{j}\right], s_{i}\right) \quad$ (because $B E F\left(a_{k}, \operatorname{pos}\left[\omega_{j}\right], s_{i}\right)=$ $\left.C N T\left(a_{k}, s_{i}\right)-A F T\left(a_{k}, \operatorname{pos}\left[\omega_{j}\right], s_{i}\right)\right)$. The actor of choice $a_{c}$ fires $A F T\left(a_{c}, \operatorname{pos}\left[\omega_{j}\right], s_{i}\right)$ times after decision state $\omega_{j}$ and the number of tokens in $c_{i . a_{c} \omega_{j}}$ returns to $A F T\left(a_{c}, \operatorname{pos}\left[\omega_{j}\right], s_{i}\right)$. These initial token resettings at the end of the PSOS iteration ensure the periodic behavior for the added components in each decision state. Thus, in a decision state only the actor of choice (which is in line with the given schedule) amongst all opponent actors of the decision state can fire and this eliminates any non-determinism because of the decision state.

The following theorems state the correctness of DSM in modeling a single PSOS for a sub-set of the actors of the SDFG.

Theorem 1. Consider PSOS $s_{i}$ as a schedule for actors $A_{i} \subseteq$ A from $\operatorname{SDFG} G(A, C)$. Assume $G^{\prime}\left(A^{\prime}, C^{\prime}\right)$ is the $S D F G$ that models $s_{i}$ in $G$ using DSM. For any execution $\sigma^{\prime}$ of $G^{\prime}\left(A^{\prime}, C^{\prime}\right)$ it holds that $\sigma$ satisfies $s_{i}$ where it is assumed that $\sigma$ is the execution of $G(A, C)$ with orderList $(\sigma, A)=$ $\operatorname{orderList}\left(\sigma^{\prime}, A\right)$.

Proof. Proposition 6 states that in a decision state of $s_{i}$, an enabled actor of the decision state which is in line with $s_{i}$ is the only actor able to fire in that state among all enabled actors in $A_{i}$. So, the order of $s_{i}$ is the only possible order of actor firing for those actors of $G^{\prime}$ in the set $A_{i}$. Proposition 2 implies that the next PSOS iteration cannot interfere. Hence, for any execution $\sigma^{\prime}$ of $G^{\prime}\left(A^{\prime}, C^{\prime}\right)$, order List $\left(\sigma^{\prime}, A_{i}\right)$ has the form of $\left(s_{i}\right)^{\kappa}$ where $\kappa \in \mathbb{N}$ (i.e., infinite repetition of $s_{i}$ ). It is assumed that $\operatorname{order} \operatorname{List}(\sigma, A)=\operatorname{order} \operatorname{List}\left(\sigma^{\prime}, A\right)$; as $A_{i} \subseteq A$, we can conclude that $\operatorname{orderList}\left(\sigma, A_{i}\right)=\operatorname{orderList}\left(\sigma^{\prime}, A_{i}\right)$. Hence, $\operatorname{order} \operatorname{List}\left(\sigma, A_{i}\right)$ also has the form of $\left(s_{i}\right)^{\kappa}$ and this form satisfies $s_{i}$; in other words, $\sigma$ satisfies $s_{i}$.

Theorem 2. Consider PSOS $s_{i}$ as a schedule for actors $A_{i} \subseteq A$ from $\operatorname{SDFG} G(A, C)$. Assume $G^{\prime}\left(A^{\prime}, C^{\prime}\right)$ is the $S D F G$ that models $s_{i}$ in $G$ using DSM. For any execution $\sigma$ of $G$ that satisfies $s_{i}$ it holds that there is exactly one $\sigma^{\prime}$ that is an execution of $G^{\prime}\left(A^{\prime}, C^{\prime}\right)$ such that $\operatorname{order} \operatorname{List}(\sigma, A)=$ $\operatorname{order} \operatorname{List}\left(\sigma^{\prime}, A\right)$.

Proof. It is assumed that the firing order of actors belonging to the set $A$ in execution $\sigma^{\prime}$ has the same actor firing order as in execution $\sigma$ and execution $\sigma$ satisfies $s_{i}$. We should show that there is precisely one execution with the property of execution $\sigma^{\prime}$ and that is a valid execution for $G^{\prime}$. In a precise way, the actor firing order related to the set $A$ in execution $\sigma$ is a possible actor firing order for the original actors (i.e., actors not added by DSM) of $G^{\prime}$ when $\sigma^{\prime}$ is an execution of $G^{\prime}$. Actor $a_{x}$ from $\operatorname{order} \operatorname{List}(\sigma, A)$ belongs either to $A_{i}$ or to $A_{o}=A \backslash A_{i}(x \in \mathbb{N})$. The state transition $\omega_{x} \stackrel{a_{x}}{\longrightarrow} \omega_{x+1}$ in execution $\sigma$ is related to the firing of $a_{x}$. The state transition $\omega_{y}^{\prime} \stackrel{a_{y}}{\longrightarrow} \omega_{y+1}^{\prime}$ in execution $\sigma^{\prime}$ is related to the firing of $a_{y}$. The difference between states from execution $\sigma$ and $\sigma^{\prime}$ is only in the extra channels added by DSM (i.e., $C_{s_{i}}$ ). Any channel from $C_{s_{i}}$ is connected to an actor from $A_{i}$; in other words, it is not connected to any actor from $A_{o}$. Incoming channels of an actor 
determine whether that actor can fire or not. Consider $\omega_{y}^{\prime}$ from execution $\sigma^{\prime}$ has the same content of state $\omega_{x}$ from execution $\sigma$ for all channels in $C$. Hence, each $a_{x} \in A_{o}$ that fires in $\omega_{x}$ of execution $\sigma$ can also fire in $\omega_{y}^{\prime}$ of execution $\sigma^{\prime}$ (i.e., $a_{y}$ is $a_{x}$ ) because the content of the state related to channels $C_{s_{i}}$ has no influence on actor enabling for any actor from $A_{o}$. But, when an actor $a_{x}$ belongs to $A_{i}$, the content of the state related to channels $C^{\prime}$ could manipulate the actor firing order. It is assumed that firings of actor $a_{x}$ in execution $\sigma$ satisfy the PSOS $s_{i}$; actor $a_{x}$ can also fire in the corresponding state (i.e., $\omega_{y}^{\prime}$ ) of execution $\sigma^{\prime}$ (i.e., $a_{y}$ is $a_{x}$ ) because components added by DSM force the firing of the actor which is in line with the given $s_{i}$ (i.e., $a_{x}$ ) among all actors in $A_{i}$ (see Proposition 6). So, the firing order of actors from $A^{\prime} \backslash A_{s_{i}}$ in execution $\sigma^{\prime}$ follows the same firing order as it is indicated in execution $\sigma$. Each actor $a \in A_{s_{i}}$ also has a single possible firing order in each PSOS iteration; if $a$ is added to control the actor firing in a decision state, it fires before the actor of choice in the decision state (see Proposition 6) and if it is added for the interiteration prevention, it fires at the end of the PSOS iteration (see Proposition 2). Hence, there exists only one possible firing order in execution $\sigma^{\prime}$ for each actor $a \in A_{s_{i}}$. So, all actors from $A^{\prime}$ have exactly one firing order in execution $\sigma^{\prime}$ where $\operatorname{order} \operatorname{List}(\sigma, A)=\operatorname{orderList}\left(\sigma^{\prime}, A\right)$.

The size of the schedule-extended graph (e.g., $G^{\prime}$ ) is dependent on the number of decision states found in the given schedule (e.g., $s_{i}$ ). In this section, decision state identification for a sub-set of actors of $\mathrm{G}$ (i.e., $A_{i} \subseteq A$ ) which belong to the schedule of interest (i.e., $s_{i}$ ) is explained regardless of existing other schedules for the rest of the actors in the SDFG (i.e., actors in $A_{o}=A \backslash A_{i}$ ). In our implementation, we consider other possible schedules designated for the rest of the actors (i.e., actors in $A_{o}$ ) to reduce the number of the decision states and as a result the size of the schedule-extended graph. As we explained in Section V-E2, actors which do not belong to $s_{i}$ should fire according to their schedule (if there is any) to perform their maximal execution. Firing of any actor $a_{o} \in A_{o}$, which belongs to another PSOS $s_{j}(j \neq i)$, in function maxExec of the DSM algorithm should satisfy the schedule to which $a_{o}$ belongs. In other words, $a_{0}$ in function maxExec of DSM fires if (1) $a_{o}$ is enabled and (2) its firing satisfies $s_{j}$. Without the second condition, firing of $a_{o}$ could enable an actor from $s_{i}$ and lead to unnecessary decision states. So, considering other schedules in the function maxExec of DSM algorithm removes such redundant decision states.

\section{APPENDIX B \\ CORRECTNESS OF SASM}

This section discusses the correctness of SASM in modeling a periodic static-order SAS for a sub-set of the actors of the SDFG. We can model multiple SASs by applying the algorithm multiple times.

SASM adds some actors, denoted by $A_{s_{i}}$, and channels, denoted by $C_{s_{i}}$ to model the SAS $s_{i}$ in SDFG $G(A, C)$. $G^{\prime}\left(A^{\prime}, C^{\prime}\right)$ is the SDFG that models $s_{i}$ in $G$ using SASM where $A^{\prime}=A \cup A_{s_{i}}$ and $C^{\prime}=C \cup C_{s_{i}}$.
Between any (nested) LSs $\alpha_{u}$ and $\alpha_{v}$, where $v=(u$ $\bmod n)+1$, in a LS SAS $s_{i}=\left\{\left(\alpha_{1}\right)^{\beta_{1}}\left(\alpha_{2}\right)^{\beta_{2}} \ldots\left(\alpha_{n}\right)^{\beta_{n}}\right\}$, SASM adds one counter actor $a_{c n t_{u}}$ and two channels $c_{c n t_{u}}$ and $c_{l_{i m}}$. The following propositions shows how these components control the actor firings in $\alpha_{u}$ and $\alpha_{v}$.

Proposition 7. For any (nested) LSs $\alpha_{u}$ and $\alpha_{v}$, where $v=$ $(u \bmod n)+1$, in $L S s_{i}=\left\{\left(\alpha_{1}\right)^{\beta_{1}}\left(\alpha_{2}\right)^{\beta_{2}} \ldots\left(\alpha_{n}\right)^{\beta_{n}}\right\}, S A S M$ ensures that $\alpha_{v}$ can only be executed for $\beta_{v}$ times after $\alpha_{u}$ is executed for $\beta_{u}$ times.

Proof. SASM creates a dependency from the right-most actor (RMA) in $\alpha_{u}$ to the left-most actor (LMA) in $\alpha_{v}$ by adding one counter actor $a_{c n t_{u}}$ and two channels $c_{c n t_{u}}$ and $c_{l i m_{u}}$. The channel $c_{c n t_{u}}$ is placed from the RMA in $\alpha_{u}$ to $a_{c n t_{u}}$; the production rate of the RMA in $\alpha_{u}$ on channel $c_{\text {cnt }_{u}}$ is set to one and consumption rate of $a_{c n t_{u}}$ on channel $c_{c n t_{u}}$ is set to the number of times that the RMA in $\alpha_{u}$ is needed to fire in $\beta_{u}$ executions of $\alpha_{u}$. Hence, actor $a_{c n t_{u}}$ can fire once after the looped schedule $\alpha_{u}$ is executed for $\beta_{u}$ times (the $1^{\text {st }}$ behavior). The channel $c_{l i m_{u}}$ is placed from $a_{c n t_{u}}$ to the LMA in $\alpha_{v}$; the production rate of $a_{c n t_{u}}$ on channel $c_{c n t_{u}}$ is set to the number of times that the LMA in $\alpha_{v}$ is needed to be fired in $\beta_{v}$ executions of $\alpha_{v}$ and the consumption rate of the LMA in $\alpha_{v}$ on channel $c_{c n t_{u}}$ is set to one. Hence, the looped schedule $\alpha_{v}$ can be executed for $\beta_{v}$ times after one firing of $a_{c n t_{u}}$ (the $2^{\text {nd }}$ behavior). It is assumed that SASM is recursively applied to each (nested) looped schedules in $s_{i}$. Considering both of the explained $1^{\text {st }}$ and $2^{\text {nd }}$ behaviors provides that $\alpha_{v}$ can only be executed for $\beta_{v}$ times after $\alpha_{u}$ is executed for $\beta_{u}$ times. $\square$

SASM only places a specific number of initial tokens (i.e., the number of times that the LMA of $\alpha_{1}$ should fire in $\alpha_{1}{ }^{\beta_{1}}$ ) on the last limiter channel (i.e., $c_{\lim _{n}}$ ) to prevent deadlock in execution; the following proposition explains how this prevents inter-iteration execution in the schedule-extended graph.

Proposition 8. Inter-iteration execution is impossible for any actor appearing in $S A S s_{i}$ in the $S D F G G^{\prime}\left(A^{\prime}, C^{\prime}\right)$.

Proof. Considering the behavior described in Proposition 7 for all pairs of consecutive (nested) looped schedules in $s_{i}$ ensures that the next schedule iteration cannot be started before the current schedule iteration ends. The initial token placed on the last limiter channel (i.e., $c_{l i m_{n}}$ ) only provides tokens for $\beta_{1}$ executions of the looped schedule $\alpha_{1}$; the executions of $\alpha_{1}$ related to the current schedule iteration consumes all tokens on the last limiter channel and this prevents the next iteration from being executed until completion of the current iteration (i.e., $\alpha_{n}$ is executed for $\beta_{n}$ times).

The following theorems state the correctness of SASM. These theorems are similar to the theorems given in Appendix VII.

Theorem 3. Consider SAS $s_{i}=\left\{\left(\alpha_{1}\right)^{\beta_{1}}\left(\alpha_{2}\right)^{\beta_{2}} \ldots\left(\alpha_{n}\right)^{\beta_{n}}\right\}$ as a schedule for actors $A_{i} \subseteq A$ from $S D F G G(A, C)$. Assume $G^{\prime}\left(A^{\prime}, C^{\prime}\right)$ is the SDFG that models $s_{i}$ in $G$ using SASM. For any execution $\sigma^{\prime}$ of $G^{\prime}\left(A^{\prime}, C^{\prime}\right)$ it holds that $\sigma$ satisfies $s_{i}$ where it is assumed that $\sigma$ is the execution of $G(A, C)$ with $\operatorname{order} \operatorname{List}(\sigma, A)=\operatorname{order} \operatorname{List}\left(\sigma^{\prime}, A\right)$. 
Proof. Considering Proposition 7 for all pairs of consecutive (nested) LSs in $s_{i}$ guarantees that the order specified in $s_{i}$ is the only possible order of actor firing for those actors of the SDFG $G^{\prime}$ in the set $A_{i}$. Proposition 8 implies that subsequent SAS iterations cannot interfere. Hence, for any execution $\sigma^{\prime}$ of SDFG $G^{\prime}\left(A^{\prime}, C^{\prime}\right)$, orderList $\left(\sigma^{\prime}, A_{i}\right)$ has the form of $\left(s_{i}\right)^{\kappa}$ where $\kappa \in \mathbb{N}$ (i.e., infinite repetition of $s_{i}$ ). It is assumed that $\operatorname{orderList}(\sigma, A)=\operatorname{order} \operatorname{List}\left(\sigma^{\prime}, A\right)$; as $A_{i} \subseteq A$, we can conclude that $\operatorname{orderList}\left(\sigma, A_{i}\right)=\operatorname{orderList}\left(\sigma^{\prime}, A_{i}\right)$. Hence, $\operatorname{order} \operatorname{List}\left(\sigma, A_{i}\right)$ also has the form of $\left(s_{i}\right)^{\kappa}$ and this form satisfies $s_{i}$; in other words, $\sigma$ satisfies $s_{i}$.

Theorem 4. Consider $S A S s_{i}$ as a schedule for actors $A_{i} \subseteq A$ from SDFG $G(A, C)$. Assume $G^{\prime}\left(A^{\prime}, C^{\prime}\right)$ is the $S D F G$ that models $s_{i}$ in $G$ using SASM. For any execution $\sigma$ of $G(A, C)$ that satisfies $s_{i}$ it holds that there is exactly one $\sigma^{\prime}$ that is an execution of $G^{\prime}\left(A^{\prime}, C^{\prime}\right)$ such that orderList $(\sigma, A)=$ $\operatorname{orderList}\left(\sigma^{\prime}, A\right)$.

Proof. It is assumed that the firing order of actors belonging to the set $A$ in execution $\sigma^{\prime}$ is the same as in execution $\sigma$ and $\sigma$ satisfies $s_{i}$. We should show that there is precisely one execution with the property of execution $\sigma^{\prime}$ and that is a valid execution for $G^{\prime}$. The actor firing order related to the set $A$ in execution $\sigma$ (i.e., $\operatorname{order} \operatorname{List}(\sigma, A)$ ) is a possible actor firing order for the original actors (i.e., actors not added by SASM) of $G^{\prime}$ when $\sigma^{\prime}$ is an execution of $G^{\prime}$. Actor $a_{x}$ from $\operatorname{orderList}(\sigma, A)$ belongs either to $A_{i}$ or to $A_{o}=A \backslash A_{i}$ $(x \in \mathbb{N})$. The state transition $\omega_{x} \stackrel{a_{x}}{\longrightarrow} \omega_{x+1}$ in execution $\sigma$ is related to the firing of $a_{x}$. The state transition $\omega_{y}^{\prime} \stackrel{a_{y}}{\longrightarrow} \omega_{y+1}^{\prime}$ in execution $\sigma^{\prime}$ is related to the firing of $a_{y}$. The difference between states from execution $\sigma$ and $\sigma^{\prime}$ is only in the channels added by SASM (i.e., $C_{s_{i}}$ ). Any channel from $C_{s_{i}}$ is connected to an actor from $A_{i}$; in other words, it is not connected to any actor from $A_{o}$. Incoming channels of an actor determine whether that actor can fire or not. Consider state $\omega_{y}^{\prime}$ from execution $\sigma^{\prime}$ has the same content of state $\omega_{x}$ from execution $\sigma$ for all channels in $C$. Hence, each $a_{x} \in A_{o}$ that fires in $\omega_{x}$ of execution $\sigma$ can also fire in $\omega_{y}^{\prime}$ of execution $\sigma^{\prime}$ (i.e., $a_{y}$ is $a_{x}$ ) because the content of the state related to channels $C_{S_{i}}$ has no influence on actor enabling for any actor from $A_{o}$. But, when an actor $a_{x}$ belongs to $A_{i}$, the content of the state related to channels $C^{\prime}$ could manipulate the actor firing order. As we assume firings of $a_{x}$ in execution $\sigma$ satisfy $s_{i}$, $a_{x}$ can fire in the corresponding state (i.e., $\omega_{y}^{\prime}$ ) of execution $\sigma^{\prime}$ (i.e., $a_{y}$ is $a_{x}$ ) because components added by SASM force the firing of the actor which is in line with the given SAS $s_{i}$ among all actors in $A_{i}$ (considering Proposition 7 for all pairs of consecutive nested LSs) and it is assumed that $a_{x}$ is in line with $s_{i}$. So, the firing order of actors from $A^{\prime} \backslash A_{s_{i}}$ in execution $\sigma^{\prime}$ follows the same firing order as indicated in $\sigma$. Each counter actor $a_{c n t_{x}} \in A_{s_{i}}$ also has a single possible firing order in each schedule iteration; it fires once after the LS $\alpha_{x}$ executed for $\beta_{x}$ times (see proof of Proposition 7). Hence, there exists only one possible firing order in $\sigma^{\prime}$ for each actor $a \in A_{s_{i}}$. So, all actors from $A^{\prime}$ have exactly one firing order in $\sigma^{\prime}$ where $\operatorname{orderList}(\sigma, A)=\operatorname{order} \operatorname{List}\left(\sigma^{\prime}, A\right)$. 